\title{
The Three Worlds of Penrose: Strings or Rotating Vectors?
}

\author{
Sebastiano Tosto \\ ENEA Casaccia, Roma, Italy \\ Email: stosto@inwind.it, stosto44@gmail.com
}

How to cite this paper: Tosto, S. (2020) The Three Worlds of Penrose: Strings or Rotating Vectors?. Journal of Applied Mathematics and Physics, 8, 1665-1705. https://doi.org/10.4236/jamp.2020.89127

Received: April 14, 2020

Accepted: August 29, 2020

Published: September 2, 2020

Copyright $\odot 2020$ by author(s) and Scientific Research Publishing Inc. This work is licensed under the Creative Commons Attribution International License (CC BY 4.0).

http://creativecommons.org/licenses/by/4.0/

\begin{abstract}
The paper examines some basic concepts of the quantum theory. It is concluded that through the concepts of rotating vectors in the complex plane and statistical formulation of quantum uncertainty the wave function $\psi$ has its own well defined physical meaning. The approach of the present paper evidences once more the tight link between quantum theory and relativistic theory.
\end{abstract}

\section{Keywords}

Corpuscular Quantum Physics, Wave Quantum Physics, Relativity

\section{Introduction}

Penrose wondered about the ability of theoretical models of describing the universe through numbers as if the mathematics was already there, whereas Wigner questioned about the unreasonable effectiveness of mathematics in physical sciences; eventually, quoting Einstein, how can it be that mathematics, being after all a product of human thought which is independent of experience, is so admirably appropriate to the objects of reality? It is surprising the fact that just three brilliant minds to whom we owe crucial contributions to the advancement of science agreed about the necessity of explaining the success of their own work [1] [2].

Penrose, in particular, identified the three fundamental steps of any science, i.e. hypothesis, observation, validation; accordingly, with reference to three ideal worlds early sketched in [3]. He postulated the existence of:

- an ideal and perfect world, timeless and reminiscent of that hypothesized by Plato, exists beyond our real and imperfect world whereas visible things of the physical reality are copies of non-visible perfect things of the ideal world; 
- a mental world, which governs our consciousness;

- a physical world, formed by the physical reality of things accessible to Science.

According to Penrose's idea an interaction exists between world 1 and world 3, i.e. the former obeys physical laws which are objects of the latter [4]. The connection between these worlds is intuitive, acknowledging the equivalence of Platonic and mathematical worlds, linked by the mathematical models via physical theories; mental and physical worlds are linked by the observation process, i.e. the measurement act, whereas mathematical and mental worlds consist of hypotheses aimed to guess the perfection of Plato's ideas from the imperfect reality of things. Also, the mental world is enclosed in a small section of the physical world: in short, our consciousness of part of the physical reality we understand is a real object that in turn highlights the structure of the Platonic world. This cyclic connection is powered by mathematics, thanks to which we extrapolate the part of physical world accessible to our consciousness up to the Platonic world. This has to do with Popper's three worlds [5], where the Platonic world consists of human concepts and abstractions, the physical world is that of matter and energy, the mental world is the individual perception and interpretation of the physical world.

Omitting philosophical considerations in this respect, try to examine the possible link between these concepts just sketched and the essential features of current physical theories. The following remarks aim to verify whether or not the physical theories fit indeed the three world scheme proposed by Penrose and Popper.

1) The quantum theory requires that the physical properties are actually determined by the experiment; in other words, the experiment turns the unknowable essence of the elusive quantum world into the knowable reality of the physical world we in fact know. This last step explains why the Penrose mental world is entitled to include the subtle quantum world. The link with the physical world is the perturbation induced by the observer during the measurement process; accordingly, the real world is a figurative representation of the mental world forcedly upset by the attempt of inspecting its pre-existing hidden reality. Einstein's claim "the moon does exist even though nobody observes it" compels thinking that "moon" is simply what we observe after having interacted with it during the measurement process, regardless of what else it might actually be if never observed. Hence the experiment turns the unknowable essence of the Plato quantum world into the knowable reality of the physical world: this last step explains why the Penrose mental world is entitled to include the subtle quantum world.

2) The determinism of the classical physics is unable to explain why the formulas, a creation of the human mind, can be fingerprint of the reality around us; an analogous difficulty follows about the epistemological meaning of scientific culture, being the mental tool we use to understand this reality rigidly fixed by 
appropriate boundary initial conditions. The answer to the previous questions is fairly simpler considering instead the quantum world: its non-locality and non-reality appear weird if referred to the common sense of the classical physics, but actually they are rational consequences of the faintish and abstract meaning of uncertainty.

3) Eventually the relativity enters into the scheme thanks to its double link with the quantum and classical worlds. In effect the relativity, at least as early formulated by Einstein, is basically classical physics extraordinarily enriched by the concept of $4 \mathrm{D}$ covariance and finite light speed; yet it is connected to the mental and physical worlds via the respective limits $\hbar \rightarrow 0$ and $c \rightarrow \infty$ from the quantum and relativistic sides [6].

The three worlds are thus tentatively linked as in Figure 1 on the basis of the aforesaid points. Penrose worried that the weak point of his scheme is the just link between abstract ideas and matter, despite His ideas are certainly amazing [7]: the special and general relativity is the large, the quantum theory is the small, both are deep-rooted in the abstract concept of human mind. Yet the considerations of the present paper shed some light in this respect via the agnostic yet heuristic concept of space-time uncertainty underlying the physical reality around us.

The present paper summarizes also some ideas already published elsewhere in order to make the text as self-contained as possible.

\section{Wave and Corpuscular Quantum Mechanics}

Several papers early published [8] [9] have shown the chance of describing the quantum systems via the statistical formulation of uncertainty only

$$
\delta p \delta x=n \hbar=\delta \varepsilon \delta t, \quad n=\text { integer },
$$

with $n$ arbitrary integer. In turn, this equation is consequence of the operative definition of space time [10]

$$
\hbar G / c^{2}
$$

further remarks and way to infer (2.1) from (2.2) are omitted here for brevity,

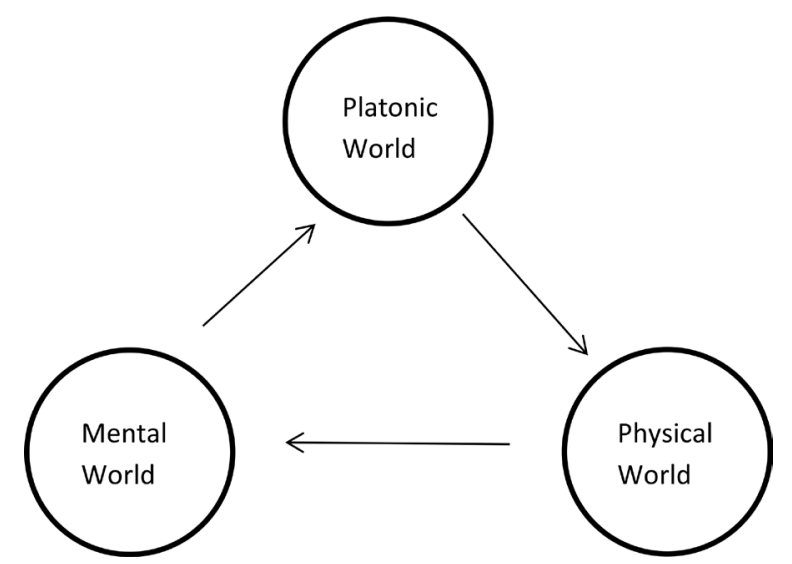

Figure 1. Penrose worlds and their possible connections with worlds of physics. 
being not essential for the purposes of the present paper. It is worth noticing instead that the product of range sizes $\delta x \delta p$ should be actually written $\delta x \cdot \delta p$, where the scalar consists of an arbitrary number of space coordinates; this remark is of interest for the string theory [11] [12], yet this heuristic implication of (2.1) is waived in the following theoretical frame. Considering one space dimension only simplifies the exposition of the model without loss of generality as concerns the purposes of the paper; even the formal notation is simplified writing throughout simply $\delta p$ instead of $\delta p_{x}$, in principle more correct. Is here interesting the fact that this equation, regarded as a fundamental postulate, splits into two equations:

$$
2 \pi \delta x=\frac{n h}{\delta p}=\frac{n h}{p-p_{0}}, \quad n \frac{\hbar}{\delta t}=\delta \varepsilon=\varepsilon-\varepsilon_{0} .
$$

Rewriting identically the first equation as follows

$$
2 \pi \delta x=n \ell, \quad \ell=\frac{h}{\delta p},
$$

one obtains

$$
\delta p=p-p_{0}=\frac{h}{\ell} .
$$

Obviously $p_{0}$ and $\varepsilon_{0}$ are two arbitrary constants that specify the initial boundary conditions of the concerned problem. At this point the reasoning is concluded with a trivial rewriting of the starting Equation (2.1), in principle nothing compels further considerations.

However is possible a particular interpretation of the last two equations.

If one regards $\delta t^{-1}$ as a frequency $\omega$, then this position brings straightforwardly into the domain of quantum mechanics, because this formalism implemented for $\delta \varepsilon$ must hold by consequence also for $\delta p$; in other words likewise as $\delta t^{-1} \rightarrow \omega$, also $\ell \rightarrow \lambda$ since frequency implies coherently wavelength. Then it is required

$$
2 \pi \delta x=n \lambda, \quad n \hbar \omega=\varepsilon-\varepsilon_{0}, \quad p-p_{0}=\frac{h}{\lambda},
$$

i.e. the elementary length $\ell$ to be repeated $n$ times along a circumference of radius $\delta x$ is actually an elementary wavelength $\lambda$. These three results do not need further explanation, they have marked the turning point from classical to quantum world; the third equation, in particular, is well known in the form $(h / 2 \pi)(2 \pi / \lambda)=\hbar \kappa$ where $\kappa$ is the so called wave vector.

Historically, according to the formulation of the quantum mechanics in its early days, the hypotheses of Planck and De Broglie were consequential after the black body theoretical model and two slit electron diffraction experiment, here these hypotheses turn to be contextual corollaries of (2.1): accepting the former compels accepting the latter as well. The conclusion is that entering the world of Planck requires the pilot waves of De Broglie, otherwise fails the logical correlation of energy and momentum: in both definitions the mass is no longer expli- 
citly evident, being instead "embedded" in the physical dimensions of $\hbar$, i.e. the corpuscle is no longer a body of matter but rather it turns to be a wave. All of this is certainly not trivial like the mere ways (2.5) of rewriting (2.1), which indeed read

$$
\delta p=n \hbar \kappa, \quad \delta \varepsilon=n \hbar \omega, \quad \kappa=\frac{1}{\delta x}, \quad \omega=\frac{1}{\delta t} .
$$

Since by definition $\delta p=p-p_{0}$ and $\delta \varepsilon=\varepsilon-\varepsilon_{0}$, in fact the second and third (2.5) read $\varepsilon=\delta \varepsilon_{\text {quant }}+\varepsilon_{0}$ and $p=\delta p_{\text {quant }}+p_{0}$, where the lower boundaries $\varepsilon_{0}$ and $p_{0}$ of the energy and momentum ranges are recognizable as initial boundary conditions of any dynamical problem of classical physics; here, however, both are completely unknown and unknowable in principle. Indeed, in the present way of thinking all uncertainty ranges hitherto introduced are arbitrary and unknowable themselves by definition of quantum uncertainty; moreover the limit $\delta x \rightarrow 0$ is in principle admissible whereas $\delta x=0$ does not, as it would imply the vanishing of uncertainty ranges compliant with the lack of determinism in the quantum world. So standard algebraic manipulations handle the ranges likewise any finite physical quantity: the uncertainty ranges, and not the random values of the respective dynamical variables, have physical meaning, as it has been shown in the quoted papers [8] [9]. It is easy to acknowledge that the step from (2.5) to (2.6) is not merely formal but has instead crucial conceptual significance: it rises two fundamental problems.

The first is that of understanding what actually $\omega$ means, i.e. what does in fact rotate with this frequency corresponding to the time lapse $\delta t$. Everybody has the perception of time lapse through the daily steps of life; nevertheless nobody cares about the fact that each one of these steps could be also related to some aspect of the quantum world if regarded as reciprocal frequencies, as this mental extrapolation is less intuitive than any act of practical meaning. Yet just this step seemingly innocuous links classical and quantum physics: the former introduces the frequency as time $\mathrm{e}^{-1}$ once having tacitly taken for granted the concept of time lapse, the latter introduces instead the time lapse as frequency ${ }^{-1}$ for reasons elucidated below. The latter position is immediately recognized thinking that quantum energy and momentum are defined by the Planck and De Broglie postulates as frequency and wavelength are wave properties of matter we are made of, whence (2.6). A crucial feature of (2.1) is that they compel considering reference values of all dynamical variables; for example $\delta p=p^{\prime}-p_{o}$ introduces the upper range boundary $p^{\prime}$ with respect to its reference value $p_{o}$, whereas $\delta \varepsilon=\varepsilon^{\prime}-\varepsilon_{o}$ regards $\varepsilon^{\prime}$ with respect to its reference value $\varepsilon_{o}$ and so on. So $p_{o} \leq p \leq p^{\prime}$ and $\varepsilon_{o} \leq \varepsilon \leq \varepsilon^{\prime}$ are random dynamical variables unknown and conceptually unknowable within the respective range sizes, unknowable themselves: in fact the Planck assumption $\delta \varepsilon=n \hbar \omega$ is immediate corollary of regarding $\delta t$ as $\omega^{-1}$ in (2.1) along with De Broglie's assumption of regarding $\delta x$ as $\lambda$. Once accepting (2.6) holds therefore the idea that a simple time lapse defines actually a corresponding quantum state characterized 
by its own energy and momentum, as it will be shown later. Moreover just the fact that (2.6) plug (2.1) into the realm of quantum world, suggests that even ignoring the steps (2.5) and (2.6), actually implied as a further aspect of (2.1), emphasizes the necessity of investigating why both are mutually consistent with the weird nature of the quantum world. Nevertheless expressing the range sizes in Planck units, as for example $\delta \varepsilon=n_{\varepsilon}^{*} \epsilon_{\text {Plank }},(2.1)$ take the unusual but crucial dimensionless form [13]

$$
n_{x}^{*} n_{p}^{*}=n=n_{\varepsilon}^{*} n_{t}^{*}
$$

where the starred numbers are real numbers completely arbitrary and unknown, whereas $n$ is an arbitrary integer; indeed by definition $\epsilon_{\text {Plank }} t_{\text {Plank }}=\hbar$. In this way the reference systems are conceptually waived in any problem where the ranges of dynamical variables replace the local dynamical variables conceptually, not as a sort of approximation; it is clear that just this conclusion and the fact that the time coordinate is inherently included together the space coordinates make the quantum uncertainty naturally compliant with the fundamental assertion of the general relativity. Hence it is in principle comprehensible why (2.1) are required and enough to bridge quantum physics and general relativity [14]. Moreover appears justified the attempt of organizing the approach of the present paper according to the ideas of wave quantum physics and corpuscular quantum physics. This distinction is really significant because (2.1) actually read

$$
\delta x \delta p=n \hbar \Leftrightarrow \delta t \delta \varepsilon=n \hbar, \quad \frac{\delta p}{\delta t}=\frac{\delta \varepsilon}{\delta x}
$$

In the first (2.8) $\hbar$ appears explicitly in both ways of expressing the quantum uncertainty of the space and time ranges, whence the chance of obtaining (2.6). In the second (2.8) $\hbar$ does not appear explicitly in the ratios of space time range sizes that clearly define the concept of force, as it is evident by dimensional reasons; in other words one could implement the second equality as it is, i.e. without necessarily knowing the existence of the Plank constant but simply accepting the physical meaning of uncertainty ranges as above defined.

The ranges of dynamical variables are well known in classical statistical theory of measurement, where these ranges are defined by the totality of random values due to unavoidable experimental errors in any measuring process. Nevertheless these classical random values are in principle known; i.e., assuming for example a Gaussian distribution law, it is possible to calculate their statistical dispersion parameters around the most probable value. In (2.1) the ranges still enclose random values of the given dynamical variable, however neither these latter nor the range sizes themselves are conceptually known and knowable; hence is unphysical the idea of calculating average values or statistical distributions of local dynamical variables. The agnostic Equation (2.1) bypasses this ignorance and clearly compels thinking an alternative way to calculate values replacing the classical concepts of statistics. The fact of neglecting anyway the local dynamical variables randomly falling in their respective ranges is crucial as concerns the link 
between quantum theory and relativity: in the first (2.8) the concept of mass can be surrogated by the physical dimensions of $\hbar$, whence the wave quantum physics; in the second (2.8) instead the mass appears explicitly through the corpuscular nature of particle, whence the corpuscular quantum physics. Although being introduced here as a postulate, (2.1) are actually corollary of a more general concept; so it is understandable why the concept of mass is inherent itself the operative definition of space time despite the physical dimensions of (2.2) are length $3 /$ time [10].

Indeed the second (2.8) reads

$$
\frac{\delta p}{v}=m=\frac{\delta \varepsilon}{v^{2}}, \quad v=\frac{\delta x}{\delta t}:
$$

once more $\delta p$ and $\delta \varepsilon$ are related likewise in (2.1), but now $n \hbar$ does no longer appear, being replaced by the proportionality factor $m$ linking $m v$ and $m v^{2}$. The connection between quantum world and classical world could not be more immediate than the one shown here after getting rid so easily of $n$ and $\hbar$. From a formal point of view, the classical physics requires neither $n \rightarrow \infty$ alone nor $\hbar \rightarrow 0$ alone but appears when these limits, which in fact exist separately, merge into a unique finite limit $m_{0} \delta x_{0}^{2} / \delta t_{0}$ dimensionally reminiscent of $\hbar$ and proportional to what we call mass $m_{0}$ through arbitrary length and time ranges. So the faintish quantum world materializes into the real tangible world we have known for centuries, with its territories and historic ages but without quantization and without $\hbar$.

From these remarks starts the discussion of the two aspects of quantum world, previously concerned by the concepts of corpuscular and wave quantum mechanics in connection with Penrose's worlds. It is interesting in this respect the chance of describing the quantum particles via an unquestionably abstract and immaterial idea: a set of two-dimensional rotating vectors in the complex plane.

\subsection{Wave Quantum Mechanics and Special Relativity}

Particularly interesting for the purposes of this subsection is the mathematical treatment exposed in [15] about the behavior of a great number $N$ statistically relevant of two-dimensional vectors rotating in a $x, y$ complex plane. Let the time evolution of these vectors be described by the function

$$
\Psi(t)=\sum_{j}^{N} \Psi_{j}(t),
$$

and let the orthogonal components of each vector be identified by the real and imaginary parts $\psi_{x}$ and $\psi_{y}$ of the complex function of modulus $\psi=|\Psi|$; then with the usual notation for these orthogonal components

$$
\psi(t)=\psi_{x}(t)+i \psi_{y}(t)=F(t) \sum_{j}^{N} \psi_{j}(t), \quad \psi_{0}=\left|\psi_{j}(t=0)\right|,
$$

where $F$ is a function to be determined. Let all vectors $\psi_{j}$ have equal modulus and be aligned at $t=0$; they are also allowed to rotate in this plane for example 
with law

$$
\psi_{j}=\psi_{x j}+i \psi_{y j}=\exp \left(i \omega_{j} t\right), \quad\left|\psi\left(t=t_{0}\right)\right|=N \psi_{0},
$$

being $\psi_{0}$ the constant modulus of each $j$-th vector and $\omega_{j}$ their arbitrary constant frequencies.

To find a reasonable form of $\psi$, which justifies by analogy that of the various $\psi_{j}$ here tentatively guessed, consider first the left hand side equality (2.10) only and define $\psi_{x}$ such that

$$
\delta \psi=\psi-\psi_{x}=i \psi_{y} ;
$$

i.e. $\psi$ and $\psi_{x}$ are the boundaries that define the range size $\delta \psi$. Noting however that also the definition $\delta \psi=\psi_{x}-\psi=-i \psi_{y}$ would have been in principle just as admissible, write then

$$
\delta \psi=\psi-\psi_{x}= \pm i \psi_{y} ;
$$

Divide now both sides of (2.12) by $\psi$ and write

$$
\frac{\delta \psi}{\psi}=1-\frac{\psi_{x}}{\psi}= \pm i \frac{\psi_{y}}{\psi}
$$

so that, since $\delta \psi / \psi=\delta \log \psi$, then

$$
\delta \log \psi=\log \psi-\log \psi_{0}=\log \frac{\psi}{\psi_{0}}= \pm i \frac{\psi_{y}}{\psi},
$$

where $\psi_{0}$ is an arbitrary constant. Then (2.13) yield

$$
\frac{\psi}{\psi_{0}}=\exp \left( \pm i \frac{\psi_{y}}{\psi}\right), \quad \frac{\psi_{x}}{\psi}=1 \mp i \frac{\psi_{y}}{\psi} .
$$

Let be in general

$$
\frac{\psi_{y}}{\psi}=\omega t+\text { const }, \quad \frac{\psi_{x}}{\psi}=1 \mp i(\omega t+\text { const }),
$$

where $\omega$ is a constant frequency. Regard therefore the first equation as series expansion of the time function $\psi_{y} / \psi=$ const + const $^{\prime} t+\cdots$ truncated at the first order; then

$$
\frac{\psi}{\psi_{0}}=\exp ( \pm i \omega t)
$$

and thus

$$
\psi=\exp ( \pm i \omega \delta t), \quad \psi_{0}=\exp \left(\mp i \omega t_{0}-\mp i c o n s t\right), \quad \delta t=t-t_{0}
$$

whence

$$
\psi_{y}=(\omega t+c o n s t) \exp ( \pm i \omega \delta t), \quad \psi_{x}=(1 \mp i(\omega t+c o n s t)) \exp (i \omega \delta t) .
$$

This result highlights that the components (2.10) of $\psi$ are linked whatever $\omega$ might specifically be. In fact $\psi_{x}$ and $\psi_{y}$ consist of real and imaginary components, as it appears in (2.18), which however does not prevent in principle the definition (2.12); also, the exponential form (2.17) justifies that of $\psi_{j}$ in 
(2.11).

Differentiate now $\psi$ with respect to time at an arbitrary time $t_{o} \geq t_{0}$; thus (2.12) and (2.15) yield at the first order according to (2.17)

$$
\begin{aligned}
\left.\delta \psi\right|_{t=t_{o}} & =\left.\frac{\delta \psi}{\delta t}\right|_{t=t_{o}}\left(t^{\prime}-t_{o}\right)= \pm\left.\psi\right|_{t=t_{o}} i \omega \delta t_{o} \\
& = \pm\left.\psi\right|_{t=t_{o}}\left( \pm \frac{i}{\hbar} \hbar \omega\right) \delta t_{o}=\left.\psi\right|_{t=t_{o}}\left( \pm \frac{i}{\hbar} \varepsilon\right) \delta t_{o}
\end{aligned}
$$

being

$$
\epsilon=\hbar \omega, \quad \delta t_{o}= \pm\left(t^{\prime}-t_{o}\right) ;
$$

in other words, $t^{\prime}$ of $\delta t_{o}$ and $\psi_{x}$ are defined as that allowing to identify $\left.\delta \psi\right|_{t=t_{0}}$ of (2.19) with $\delta \psi$ of (2.12). Merging these equations means $\left.\delta \psi\right|_{t=t_{o}}= \pm\left. i \psi_{y}\right|_{t=t_{o}}$ i.e., according to (2.19) and (2.18), $\omega \delta t_{o}= \pm \omega\left(t+t_{o} / \omega\right)$. To link this result with (2.15) and (2.17), put $t_{o}=$ constant , which is in fact possible because by definition the range sizes are arbitrary likewise as the boundary coordinates, so that

$$
t^{\prime}-t_{o}= \pm \delta t_{\omega}, \quad \delta t_{\omega}=t+\frac{\text { const }}{\omega}
$$

in principle both signs are admissible because the arbitrary $t^{\prime}$ and $t_{o}$ are consistent with both $t^{\prime}<t_{o}$ and $t^{\prime}>t_{o}$. This result is a mathematical answer to the question posed about (2.6), i.e. a frequency $\omega$ corresponds to the reciprocal time lapse $\delta t_{o}^{-1}$. In turn $\delta t_{\omega}$ can take itself both signs owing to arbitrary values of const $\gtreqless 0$.

Once more this conclusion is consistent with and required by the concept of uncertainty. On the one hand, once having introduced any range $x-x_{o}$, in principle nothing prevents putting $x_{o} \lessgtr x$; it means concerning even a range on the negative side of an $x$ axis of an arbitrary reference system, i.e. negative $x$ coordinates. On the other hand this is compliant with (2.1) implementing corresponding $\delta p \lessgtr 0$, which obviously means concerning negative components of $\boldsymbol{p}$ along the $\boldsymbol{x}$ direction. More subtle is this reasoning when one concerns the conjugate couple $\delta \varepsilon$ and $\delta t$, for which holds of course an identical reasoning: yet in this case negative range sizes imply negative energy states and negative time range, i.e. negative quantum states are in principle allowed but require time reversal. Anyway, note all arbitrary quantities appearing in (2.19) allow writing in general (2.19)

$$
\frac{\hbar}{i} \frac{\delta \psi}{\delta t}= \pm \epsilon \psi, \quad \psi=\psi_{0} \exp \left( \pm \frac{i}{\hbar} \epsilon \delta t\right), \quad \epsilon=\hbar \omega, \quad \delta t=t^{\prime}-t .
$$

The double sign, in principle not to be excluded as formerly introduced since (2.12), emphasizes that either chance allowed for $\epsilon$ depends on whether $\delta t \gtrless 0$. In other words, regardless of any relativistic assumption, (2.1) is enough to conclude that $t$ and $-t$ included in $\delta t>0$ and $\delta t<0$ are respectively compatible with states of positive and negative energy $\epsilon$ and $-\epsilon$ of the par- 
ticle; consequently, negative or positive energy states of a particle imply respective time reversal. For this reason (2.21) is identically defined by $( \pm \epsilon) \delta t$ or $\epsilon( \pm \delta t)$, i.e. positive energy state times positive time range or negative energy state times negative time range.

The result (2.21) is further extended. A relevant implication of this reasoning is that (2.16) can be identically rewritten as

$$
\frac{\psi}{\psi_{0}}=\exp \left( \pm i \omega t v_{x} / v_{x}\right)
$$

where $v_{x}$ is the $x$-component of an arbitrary velocity of modulus $v$, so, recalling (2.6),

$$
\frac{\psi}{\psi_{0}}=\exp ( \pm i \ell \kappa), \quad \kappa=\frac{\omega}{v_{x}}, \quad \ell=v_{x} t .
$$

In other words time and time ${ }^{-1}$ of (2.16) turn now into $\ell$ and $\kappa$ having physical dimensions length and length ${ }^{-1} ;$ i.e. it is possible to write

$$
\exp ( \pm i \omega t) \rightarrow \exp ( \pm i x / \lambda), \quad \lambda=\frac{v}{v},
$$

so that (2.19) turns into

$$
\begin{aligned}
& \left.\delta \psi\right|_{x=x_{o}}=\left.\frac{\delta \psi}{\delta x}\right|_{x=x_{o}} \quad\left(x^{\prime}-x_{o}\right)=\left.\psi\right|_{x_{o}} \frac{i}{\lambda} \delta x=\left.\psi\right|_{x_{o}} \frac{i \hbar}{\hbar \lambda} \delta x, \\
& \delta x=x^{\prime}-x_{o}, \quad \frac{\hbar}{i} \frac{\delta \psi}{\delta x}= \pm p \psi .
\end{aligned}
$$

Now the double sign in intuitively acknowledged, actually it is according to (2.10) the momentum component along an arbitrary $x$ axis. Eventually the last (2.23) is consistent with a result analogous to (2.21)

$$
\frac{\hbar}{i} \frac{\delta \psi}{\delta x}= \pm p \psi, \quad \psi=\psi_{0} \exp \left( \pm \frac{i}{\hbar} p \delta x\right) .
$$

So (2.21) and (2.23) show that

$$
\pm \frac{\hbar}{i} \frac{\delta}{\delta t}, \quad \mp \frac{\hbar}{i} \frac{\delta}{\delta x}
$$

are the non-relativistic operators of energy and momentum usually postulated through the position $\delta \rightarrow \partial$. Of course both $\delta$ and $\partial$ have the usual meaning of change of something as a function of something else, i.e. of time coordinate $t$ or space coordinate $x$, so the previous symbol $\rightarrow$ is justified owing to the arbitrariness of range sizes $\delta t$ and $\delta x$, which can even be thought tending to $\delta x \rightarrow 0$ and $\delta t \rightarrow 0$ as particular cases.

It is essential to remark that the signs \pm in (2.25) are not necessarily corresponding as initially introduced in the separate (2.21) and (2.24), being the former related to that of $\delta t$ the latter to the component of $v_{x}$ along $x$. Actually it is easy to realize that the signs must be just opposite, whence the notation. Indeed subtracting side by side (2.21) and (2.24) multiplied by the modulus $v$ of an arbitrary velocity one finds, depending on either correspondence of \pm signs, 


$$
\frac{\hbar}{i} \frac{\delta \psi}{\delta t}-v \frac{\hbar}{i} \frac{\delta \psi}{\delta x}=( \pm \hbar \omega-(\mp) p v) \psi= \pm(\hbar \omega+p v) \psi=\delta \varepsilon_{( \pm, \mp)} \psi
$$

or

$$
\frac{\hbar}{i} \frac{\delta \psi}{\delta t}-v \frac{\hbar}{i} \frac{\delta \psi}{\delta x}=( \pm \hbar \omega-( \pm) p v) \psi= \pm(\hbar \omega-p v) \psi=\delta \varepsilon_{( \pm, \pm)} \psi .
$$

The left hand side reads in both cases $\epsilon_{2}-\epsilon_{1}$ by dimensional reasons, i.e. it represents just the energy range that however appears in turn at the right hand side as $\delta \varepsilon_{( \pm, \mp)}$ or $\delta \varepsilon_{( \pm, \pm)}$depending on either choice of signs. Yet the latter cannot be regarded as an uncertainty range, because a value of $v=\hbar \omega / p$ in principle exists such that $\delta \varepsilon_{( \pm, \pm)}=0$; but a null range is inconsistent with the concept of uncertainty. Hence the former position ensuring $\delta \varepsilon_{( \pm, \mp)} \neq 0$ is the correct one.

Eventually it is also worth remarking that in principle are also admissible $\delta x \rightarrow \infty$ and $\delta t \rightarrow \infty$. This point is of interest when showing that (2.17) and (2.18) are consistent with (2.12). Indeed let us consider once more $\delta \psi$ of (2.19) at any $t$ around an arbitrary $t_{o}$; accordingly one finds via Euler's formula (2.19) and (2.15) yield

$$
i \omega \mathrm{e}^{i \omega\left(t-t_{o}\right)} \delta t=i(\omega t+c o n s t) \mathrm{e}^{i \omega\left(t-t_{o}\right)} .
$$

Equating the real and imaginary parts of this equation one finds

$$
\begin{aligned}
& \omega \sin \left(\omega\left(t-t_{o}\right)\right) \delta t=(\omega t+\text { const }) \sin \left(\omega\left(t-t_{o}\right)\right), \\
& \cos \left(\omega\left(t-t_{o}\right)\right) \omega \delta t=(\omega t+\text { const }) \cos \left(\omega\left(t-t_{o}\right)\right) ;
\end{aligned}
$$

the fact that a unique value of $\delta t$ fulfills both equations

$$
\delta t_{\omega}=\frac{\omega t+\text { const }}{\omega}
$$

i.e. just that already found in (2.20), means that regardless of the particular $t_{o}$ both real and imaginary parts of (2.27) reduce to identities with the unique $\delta t_{\omega}$ previously introduced, as it is easy to verify. In effect, with const $<0$ this definition of time range takes the expected form $\delta t_{\omega}=t-\mid$ const $\mid / \omega$ : on the one hand $t$ appears as a local time coordinate exactly as any $x$ within $\delta x$, on the other hand since in principle const $\leq 0$ it appears that $\delta t \rightarrow \infty$ for $t \rightarrow \infty$ and also $\delta t \rightarrow 0$ for $t \rightarrow$ const/ $\omega$ whatever $\omega$ might be via an appropriate value of the arbitrary const. Analogous reasoning holds for $\delta x$, but of course neither the time range size nor the space range size can vanish because it would contradict (2.1): it would require $n=0$ i.e. no allowed states at all.

Hence this approach shows why (2.21) and (2.23) link the formalism based directly on (2.1) and that based on the wave equation $\psi$; indeed the fact that $\epsilon$ and $p$ appear related to the operators (2.25) acting on $\psi$ is nothing else but the energy and momentum of a free particle expressed according to the operator formalism of wave mechanics via the complex function $\psi$.

As (2.15) shows that $\psi_{y} / \psi$ is real, write now (2.19) as 


$$
\frac{\psi_{y}}{\psi}=\frac{\omega}{\omega^{\prime}}+\text { const }^{\prime}, \quad \omega^{\prime}=\frac{1}{\delta t} .
$$

So

$$
\delta\left(\frac{\psi_{y}}{\psi}\right)=-\frac{\omega}{\omega^{\prime 2}} \delta \omega^{\prime}
$$

i.e. the ratio $\omega / \omega^{\prime}$ defines $\omega^{\prime}$ without loss of generality via (GRQ). Let be

$$
\omega^{\prime} \approx b+a\left(\omega^{\prime \prime}-\omega_{c}\right)+f\left(\omega^{\prime \prime}-\omega_{c}\right)^{2}+\cdots, \quad \delta \omega^{\prime}=\omega^{\prime \prime}-\omega_{c}
$$

the power series expansion of $\omega^{\prime}$ around an arbitrary $\omega_{c}$ with constant coefficients $a$ and $b$ and $f$, so $(2.29)$ at the first order, i.e. $f \approx 0$, reads

$$
-\delta\left(\frac{\delta \psi_{y}}{\psi}\right)=\frac{\omega \delta \omega^{\prime}}{b^{2}+a^{2}\left(\omega^{\prime \prime}-\omega_{c}\right)^{2}+2 a b\left(\omega^{\prime \prime}-\omega_{c}\right)} .
$$

If in particular

$$
a^{2}\left(\omega^{\prime \prime}-\omega_{c}\right)^{2} \gg 2 a b\left(\omega^{\prime \prime}-\omega_{c}\right) \text { i.e. } \quad a\left(\omega^{\prime \prime}-\omega_{c}\right) \gg 2 b,
$$

which is possible with an appropriate value of $\omega^{\prime \prime}$ that defines the validity of the truncation of series expansion (2.30), then

$$
-\delta\left(\frac{\psi_{y}}{\psi}\right)=\frac{\omega \delta \omega^{\prime}}{b^{2}+a^{2}\left(\omega^{\prime}-\omega_{c}\right)^{2}}=\frac{\tau^{\prime} \delta \omega^{\prime}}{1+a^{\prime}\left(\omega^{\prime}-\omega_{c}\right)^{2}}, \quad \tau^{\prime}=\frac{\omega}{b^{2}}, \quad a^{\prime}=\frac{a^{2}}{b^{2}}
$$

i.e.

$$
-\frac{\delta\left(\psi_{y} / \psi\right)}{\delta \omega^{\prime}}=\frac{\tau^{\prime}}{1+a^{\prime}\left(\omega^{\prime}-\omega_{c}\right)^{2}} .
$$

This expression holds during a time range $\delta t^{\prime} \equiv \omega^{\prime-1}$ for any $\omega^{\prime}$ around $\omega_{c}$ under the aforesaid first order approximation conditions; it is compliant with the known formula

$$
P\left(\omega^{\prime}\right)=\frac{\tau / \pi}{1+\left(\omega^{\prime}-\omega_{c}\right)^{2} \tau^{2}}, \quad \tau^{\prime} \equiv \frac{\tau}{\pi}, \quad a^{\prime} \equiv \tau^{2},
$$

which clarifies that $\omega_{c}$ is the central frequency of the frequency spectrum for an exponential decay of a system of rotating vectors of modulus $\psi_{j}$ with average time $\tau$ i.e.

$$
P\left(\omega^{\prime}\right)=-\frac{\delta\left(\psi_{y} / \psi\right)}{\delta \omega^{\prime}} .
$$

On the one hand this clarifies the physical meaning of $\psi_{y}$. On the other hand (2.17) allows writing the analytical form of $\psi_{j}$ guessed in (2.11) for the $j$-th vector rotating in the complex plane with constant frequency $\omega_{j}$. The vectors aligned at $t=t_{0}$ as in (2.11) turn into misaligned vectors at $t>t_{0}$ since $\omega_{j}$ are in general all different.

It appears immediately clear in this reasoning that the driving force of this misalignment should by the entropy increase of the system described in the 
second equality (2.10) and (2.11). This relevant point concerning the link between wave mechanics and second law of thermodynamics deserves being further highlighted, as it involves indeed a fundamental principle of Nature.

Consider to this purpose the second equality (2.10); owing to (2.16) and (2.11) it reads

$$
F=\frac{\psi_{0}}{\sum_{j} \exp \left(i t \delta \omega_{j}\right)}, \quad \delta \omega_{j}=\omega_{j}-\omega .
$$

Introduce now a new complex function $S^{*}$ such that

$$
\begin{aligned}
& S^{*}=-\log \left(\frac{F}{\psi_{0}}\right)=\log \sum_{j}^{N} \exp \left(i t \delta \omega_{j}\right), \\
& \delta S^{*}=\frac{\sum_{j}^{N} i \delta \omega_{j} \exp \left(i t \delta \omega_{j}\right)}{\sum_{j}^{N} \exp \left(i t \delta \omega_{j}\right)} \delta t,
\end{aligned}
$$

according to which

$$
\frac{\delta S^{*}}{\delta t}=\frac{\sum_{j}^{N} i \delta \omega_{j} \exp \left(i t \delta \omega_{j}\right)}{\sum_{j}^{N} \exp \left(i t \delta \omega_{j}\right)}
$$

this result defines $F$ a constant $\log \left(\psi_{0}\right)$ apart. Thus, via Euler's formula,

$$
\frac{\delta S^{*}}{\delta t}=\frac{i \sum_{j=1}^{N} \delta \omega_{j} \cos \left(t \delta \omega_{j}\right)-\sum_{j=1}^{N} \delta \omega_{j} \sin \left(t \delta \omega_{j}\right)}{\sum_{j=1}^{N} \cos \left(t \delta \omega_{j}\right)+i \sum_{j=1}^{N} \sin \left(t \delta \omega_{j}\right)} .
$$

A glance to this equation suggests that multiplying numerator and denominator of the second equality by

$$
\sum_{j=1}^{N} \cos \left(t \delta \omega_{j}\right)-i \sum_{j=1}^{N} \sin \left(t \delta \omega_{j}\right)
$$

it takes the form $a+i b$; so it is easy to calculate the real and imaginary parts of the resulting equation, which read respectively

$\Re\left(\frac{\delta S^{*}}{\delta t}\right)=-\frac{\sum_{j=1}^{N} \delta \omega_{j} \sin \left(t \delta \omega_{j}\right) \sum_{j=1}^{N} \cos \left(t \delta \omega_{j}\right)-\sum_{j=1}^{N} \delta \omega_{j} \cos \left(t \delta \omega_{j}\right) \sum_{j=1}^{N} \sin \left(t \delta \omega_{j}\right)}{\left(\sum_{j=1}^{N} \cos \left(t \delta \omega_{j}\right)\right)^{2}+\left(\sum_{j=1}^{N} \sin \left(t \delta \omega_{j}\right)\right)^{2}}$

and

$$
\mathfrak{J}\left(\frac{\delta S^{*}}{\delta t}\right)=\frac{\sum_{j=1}^{N} \cos \left(t \delta \omega_{j}\right) \sum_{j=1}^{N} \delta \omega_{j} \cos \left(t \delta \omega_{j}\right)+\sum_{j=1}^{N} \sin \left(t \delta \omega_{j}\right) \sum_{j=1}^{N} \delta \omega_{j} \sin \left(t \delta \omega_{j}\right)}{\left(\sum_{j=1}^{N} \cos \left(t \delta \omega_{j}\right)\right)^{2}+\left(\sum_{j=1}^{N} \sin \left(t \delta \omega_{j}\right)\right)^{2}} .
$$


Note that the denominator of both equations is surely positive, so it is interesting to examine the signs of the respective numerators.

As concerns $S^{*}$ itself it is easy to write the first $(2.32)$ as $S^{*}=s_{r e}+i s_{i m}$, whose real and imaginary parts are

$$
\mathfrak{R}\left(S^{*}\right)=\frac{1}{2} \ln \left(\left(\sum_{j=1}^{N} \cos \left(t \delta \omega_{j}\right)\right)^{2}+\left(\sum_{j=1}^{N} \sin \left(t \delta \omega_{j}\right)\right)^{2}\right)
$$

and

$$
\mathfrak{I}\left(S^{*}\right)=\arctan \left(\sum_{j=1}^{N} \sin \left(t \delta \omega_{j}\right)\left(\sum_{j=1}^{N} \cos \left(t \delta \omega_{j}\right)\right)^{-1}\right)
$$

To investigate the properties of these four equations is enough a simple assumption; write reasonably

$$
\omega_{j}=2 \pi v_{j}=2 \pi n_{j} v
$$

which follows simply from $\hbar \omega_{j}=h v_{j}=n_{j} h v$ where of course $n_{j}$ are arbitrary integers. So implement (2.36) and (2.37) along with (2.34) and (2.35) to investigate $S^{*}$ and $\delta S^{*} / \delta t$ as a function of $N$. Figure 2 and Figure 3 report the plots of $\mathfrak{R}\left(S^{*}\right)$ and $\mathfrak{R}\left(\delta S^{*} / \delta t\right)$ as a function of $N$; Figure 4 and Figure 5 report $\mathfrak{I}\left(S^{*}\right)$ and $\mathfrak{I}\left(\delta S^{*} / \delta t\right)$.

First of all simple direct calculations show that if $\omega_{j}$ are all equal to a unique value $\omega$, then for any $N$ it appears that:

(i) $\mathfrak{R}\left(\delta S^{*}\right)=0$, i.e. there is no change of $\mathfrak{R}\left(S^{*}\right)$;

(ii) $\mathfrak{R}\left(S^{*}\right)=\log (N)$;

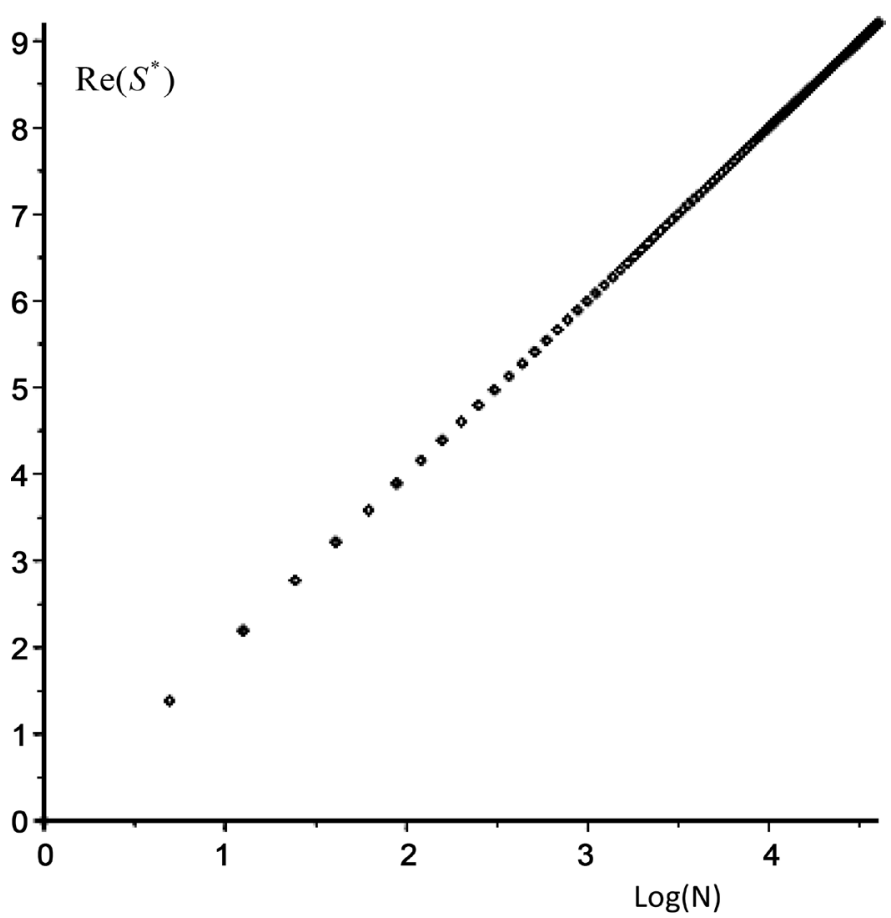

Figure 2. $\mathfrak{R}\left(S^{*}\right)$ vs $\log N$, Equation (2.36). 


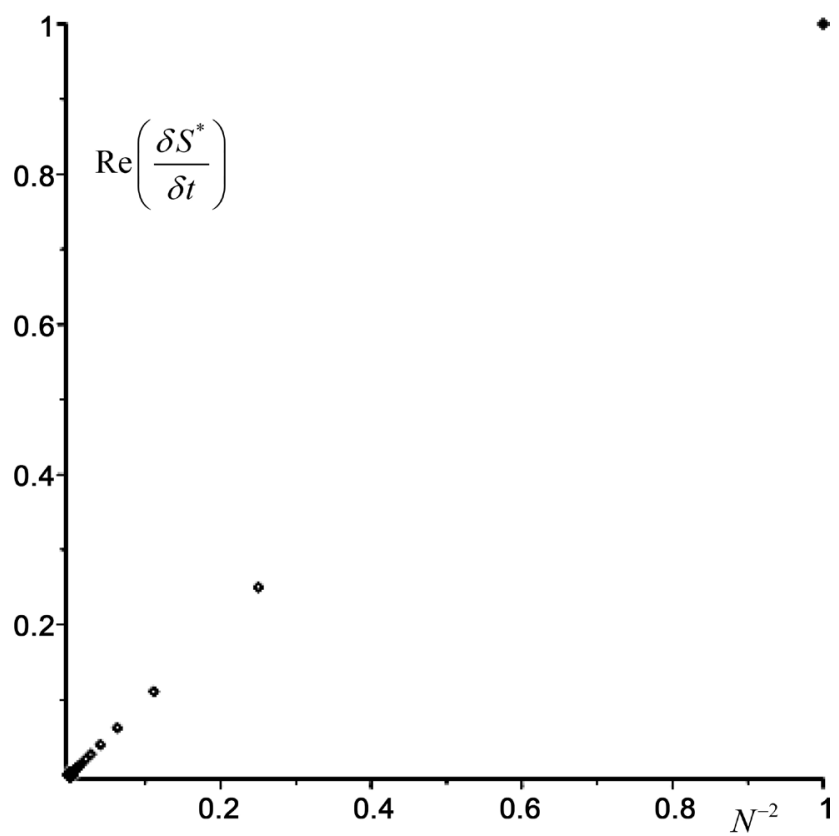

Figure 3. $\mathfrak{R}\left(\delta S^{*} / \delta t\right)$ vs $N^{-2}$, Equation (2.34).

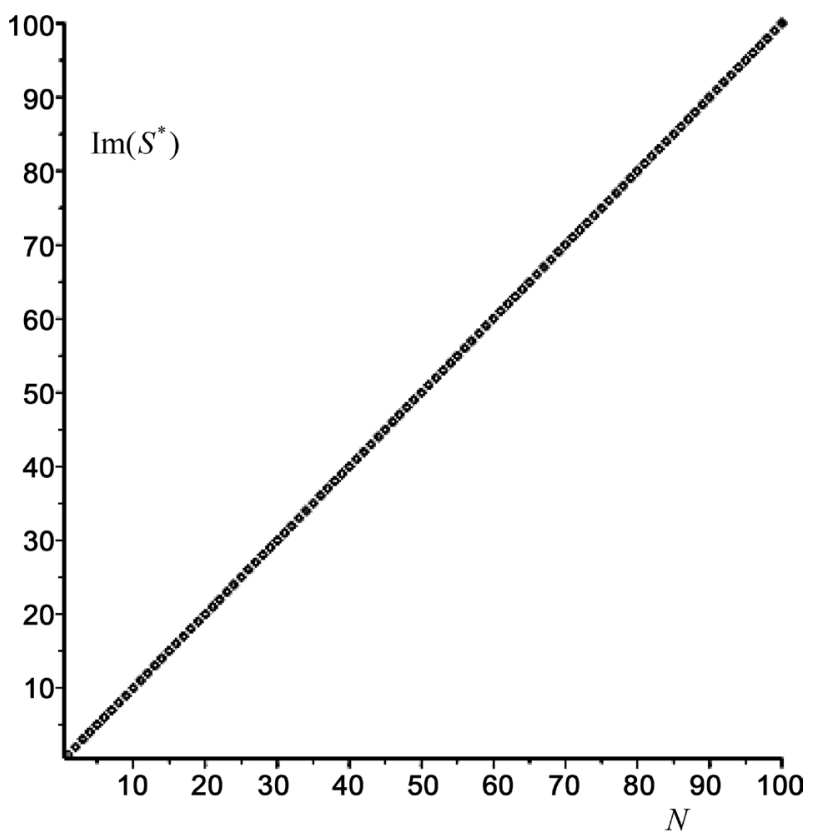

Figure 4. $\Im\left(S^{*}\right)$ vs $N$, Equation (2.37).

(iii) $\mathfrak{I}\left(S^{*}\right)=t \delta \omega$, i.e. $\mathfrak{I}\left(\delta S^{*}\right)=\delta(\omega t)$ at given $t$;

(iv) $\mathfrak{I}\left(\delta S^{*}\right)=\delta \omega$.

In other words if all vectors rotate with a unique $\omega$, then they remain aligned likewise as at $t=t_{0}$ and thus the disorder increase of the system of vectors due to their misalignment with respect to the initial configuration is null. It also appears that $\mathfrak{R}\left(S^{*}\right)$ is a logarithmic function of $N$. 


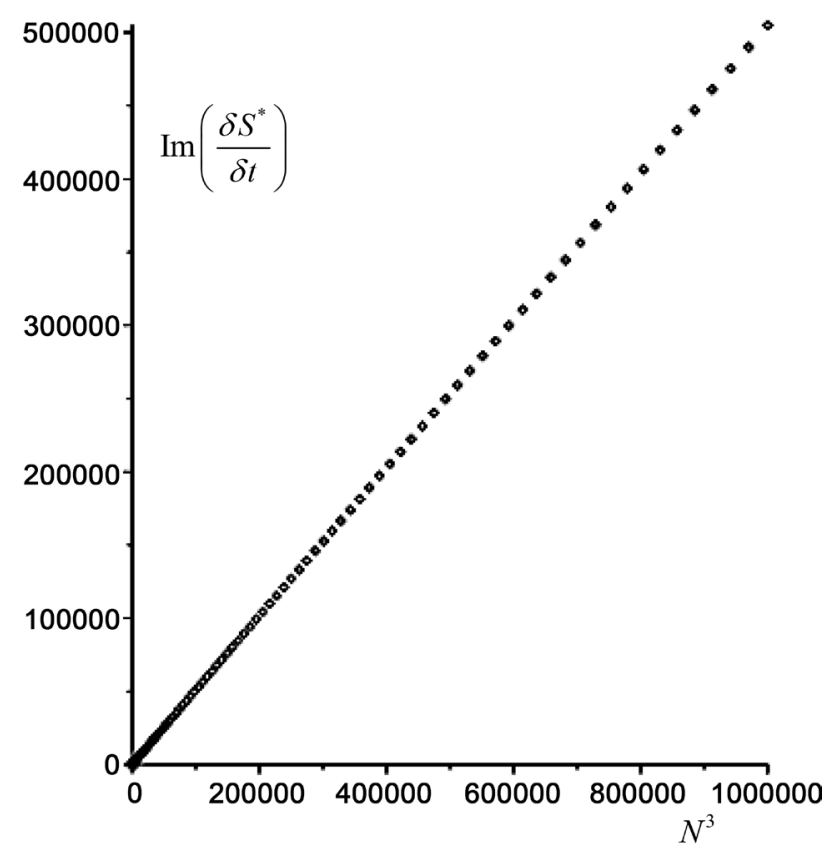

Figure 5. $\Im\left(\delta S^{*} / \delta t\right)$ vs $N^{3}$, Equation (2.35).

The general case where $\omega_{j} \neq \omega$ confirms these remarks; the plots 2 to 5 have been calculated taking arbitrarily from $N=1$ up to the maximum value $N=100$. For comparison purposes the calculations have been carried out taking advantage that linear trends are in fact obtainable in all cases.

Figure 2 shows that $\mathfrak{R}\left(S^{*}\right)$ is proportional to $\log (N)$; Figure 3 and Figure 5 show, mostly important, that both real and imaginary parts of $\delta S^{*} / \delta t$ are always positive and respectively proportional to $N^{-2}$ and $N^{3}$. Also, Figure 4 shows a linear dependence of $\mathfrak{I}\left(S^{*}\right)$ upon $N$. Since all complex rotators are in their own quantum states defined by the respective $\omega_{j}$, it follows that $S^{*}$ is an extensive property related to the disorder of the system; also $\delta S^{*} / \delta t$ increases with time because both imaginary and real parts are always positive.

These results suggest reasonably that $\mathfrak{R}\left(S^{*}\right)$ yields the dimensionless entropy $S$ that measures the degree of configuration disorder of the system of complex rotating vectors, whereas the linear proportionality of $\mathfrak{I}\left(S^{*}\right)$ suggests an energy $T S$ via the proportionality factor $T$. Figure 3 and Figure 5 show eventually that holds the requirement of spontaneous entropy increase in the isolated system of rotating vectors so far tacitly assumed. Further investigations are in progress about the aforesaid $N^{-2}$ and $N^{3}$ dependencies; assuming a cubic lattice of $N$ rotators, these dependencies suggest surface ${ }^{-1}$ and volume effects to be still identified and better understood. What is anyway remarkable even at this stage of knowledge, however, is the clear connection of the quantum results with the basic principles of thermodynamics.

Collect now together the starting Equation (2.12), (2.15), (2.14) and (2.21) in a unique chain of equations to check the self-consistency of all positions hitherto implemented; so 


$$
\frac{\delta \psi}{\delta t}=\frac{\psi-\psi_{x}}{\delta t}=i \frac{\psi_{y}}{\delta t}=i \frac{\psi_{y} \delta \varepsilon}{n \hbar}=i \psi \frac{\epsilon}{\hbar},
$$

being $\epsilon$ the eigenvalue of (2.21) in agreement with (PT2). Here the \pm signs of (2.12) are omitted assuming that they combine with the corresponding $\mp$ od $\delta t$. Hence

$$
\frac{n \hbar}{i} \frac{\delta \psi}{\delta t}=n \hbar \frac{\psi_{y}}{\delta t}=\psi_{y} \delta \varepsilon=n \epsilon \psi
$$

Then, in particular, the last equality yields

$$
\delta \varepsilon=n \epsilon \frac{\psi}{\psi_{y}}=\frac{n \epsilon}{\omega t+\text { const }} .
$$

As the time $t$ is here a dynamical variable likewise the space coordinates, i.e. it ranges in principle from 0 to $\infty$, it follows that $\delta \varepsilon=\epsilon^{\prime \prime}-\epsilon^{\prime}$ is defined from an upper boundary $\epsilon^{\prime \prime}=n \varepsilon /$ const for $t=0$ to a lower boundary $\epsilon^{\prime}=0$ for $t \rightarrow \infty$. The notation different from $\delta \varepsilon_{( \pm, \mp)}$ in (2.26) emphasizes that in fact the range sizes are conceptually arbitrary, unknown and indeterminable, whence the chance of writing identically $\epsilon^{\prime \prime}-\epsilon^{\prime}$ instead of $\epsilon_{2_{( \pm, \mp)}}-\epsilon_{1_{( \pm, \mp)}}$. Moreover, to check the connection of (2.21) with (2.1) eliminate from (2.40) $\hbar / \delta t=\delta \varepsilon / n$ and write with the help of (2.13), (2.15), (2.16) and (2.41)

$$
n i=\frac{\delta \psi}{\psi} \frac{\delta \varepsilon}{\epsilon}, \quad \frac{\delta \varepsilon}{\epsilon}=\frac{n}{\omega t+c o n s t}, \quad \frac{\delta \psi}{\psi}=i \Omega \delta t=i(\omega t+\text { const }),
$$

where

$$
\Omega=\omega \frac{\delta t_{\omega}}{\delta t}, \quad \delta t_{\omega}=t+\frac{\text { const }}{\omega}
$$

Hence (2.41) yields

$$
\frac{\delta \varepsilon}{\epsilon}=\frac{n / \omega}{\delta t_{\omega}}=\frac{\delta \varepsilon_{\omega}}{\hbar \omega}
$$

The first (2.43) means that $\hbar \omega$ is any value in $\delta \varepsilon_{\omega}$, whereas $\epsilon$ is in general any kind of energy enclosed in its corresponding $\delta \varepsilon$. Moreover merging the first and third (2.42) yields $n i=i \Omega \delta t \delta \varepsilon / \epsilon$ and thus, replacing $\delta t$ via (2.1), $n i=i \Omega(n \hbar / \delta \epsilon) \delta \varepsilon / \epsilon$; therefore as expected $\epsilon=\hbar \Omega$. Also, multiplying side by side this latter and the second (2.6), one finds

$$
\epsilon \delta \varepsilon_{\omega}=n \hbar^{2} \omega \Omega, \quad \frac{n \epsilon}{\delta t_{\omega}}=\hbar \omega \Omega
$$

On the one hand this result can be also obtained considering directly the definition of $\Omega$ in (2.46), i.e. (2.1) and (2.39) confirm the validity of (2.11). Indeed it is also possible to write (2.42) with the help of (2.19) as

$$
\begin{aligned}
& \left.\delta \psi\right|_{t}=\left.\sum_{j}^{N} i \omega_{j} \exp \left(i \omega_{j} t\right) \delta t^{\prime} \equiv \psi\right|_{t} \frac{\sum_{j}^{N} i \omega_{j} \exp \left(i \omega_{j} t\right)}{\psi_{0} \sum_{j}^{N} \exp \left(i \omega_{j} t\right)} \delta t^{\prime}, \\
& \delta t^{\prime}=t^{\prime}-t,
\end{aligned}
$$


so that comparing with (2.42) one infers

$$
i \Omega \delta t=\frac{\sum_{j}^{N} i \omega_{j} \exp \left(i \omega_{j} t\right)}{\psi_{0} \sum_{j}^{N} \exp \left(i \omega_{j} t\right)} \delta t^{\prime}=\frac{\left.\delta \psi\right|_{t}}{\left.\psi\right|_{t}}
$$

and thus

$$
\Omega=\frac{\sum_{j}^{N} \omega_{j} \exp \left(i \omega_{j} t\right)}{\psi_{0} \sum_{j}^{N} \exp \left(i \omega_{j} t\right)} \frac{\delta t^{\prime}}{\delta t} .
$$

Hence

$$
n=\frac{\sum_{j}^{N} \omega_{j} \exp \left(i \omega_{j} t\right)}{\sum_{j}^{N} \exp \left(i \omega_{j} t\right)} \delta t \frac{\delta \varepsilon}{\epsilon}=\frac{n \hbar}{\epsilon} \frac{\sum_{j}^{N} \omega_{j} \exp \left(i \omega_{j} t\right)}{\sum_{j}^{N} \exp \left(i \omega_{j} t\right)}=n \frac{\hbar \Omega}{\epsilon},
$$

i.e. $\hbar \Omega=\epsilon$; thus $\hbar \Omega$ yields the eigenvalue $\epsilon$ of the wave Equation (2.21) of the particle, as reasonably expected.

On the other hand, owing to (2.39),

$$
n=\frac{\delta \varepsilon_{\omega} \delta t_{\omega}}{\hbar}=n \frac{\hbar \Omega}{\epsilon}
$$

so that (2.6) yields

$$
\epsilon \delta \varepsilon=n \hbar^{2} \Omega \omega .
$$

Since $\Omega \omega$ is a square reciprocal time, put now

$$
\omega \Omega=\frac{1}{\tau} \delta\left(\frac{1}{t}\right)
$$

and thus

$$
\begin{aligned}
& \Omega \omega=-\frac{\delta t}{\tau t^{2}}=-\frac{\tau^{\prime \prime}}{\tau t^{2}}+\frac{\tau^{\prime}}{\tau t^{2}}=\frac{1}{t^{\prime 2}}-\frac{1}{t^{\prime \prime 2}}, \quad \delta t=\tau^{\prime \prime}-\tau^{\prime} \\
& t^{\prime 2}=\frac{\tau}{\tau^{\prime}} t^{2}, \quad t^{\prime \prime 2}=\frac{\tau}{\tau^{\prime \prime}} t^{2}
\end{aligned}
$$

where $\tau$ is a time constant added for dimensional reasons; $\tau^{\prime}$ and $\tau^{\prime \prime}$ are arbitrary time values defining the range $\delta t$, which results expressed via $t^{\prime}$ and $t^{\prime \prime}$ in a form more compact and convenient for the following reasoning. The position (2.50) has been guessed in order to express $\Omega \omega$ as a difference of square reciprocal times corresponding to the left hand side of (2.49), which actually consists of a difference of square energies as it reads $\epsilon\left(\epsilon_{2}-\epsilon_{2}\right)$ likewise as in (PT2).

Implement first (2.50) writing

$$
\frac{1}{\tau} \delta\left(\frac{1}{t}\right)=\delta\left(\frac{1}{\tau} \frac{1}{t}\right)=\delta\left(\frac{\omega}{\tau}\right)
$$

so that (2.49) reads 


$$
\begin{aligned}
\frac{1}{2} \delta\left(\epsilon^{2}\right) & =\frac{1}{2} \delta\left(\epsilon^{2}+\text { const }\right)=\delta\left(n \hbar \omega \frac{\hbar}{\tau}\right)=\delta\left(n \frac{h v}{\lambda} \frac{\hbar}{\tau}\right) \\
& =\delta\left(\frac{n h v}{\lambda} \frac{\hbar}{\lambda} \frac{\lambda}{\tau}\right)=\delta\left(p^{\prime} v^{\prime} p v\right)
\end{aligned}
$$

being obviously $\delta$ const $=0$ whereas $\lambda$, by dimensional reasons, an arbitrary wavelength; this yields

$$
\hbar \omega=h v=h \frac{v}{\lambda}, \quad p=\frac{h}{\lambda}, \quad p^{\prime}=\frac{n \hbar}{\lambda}, \quad v=\lambda v, \quad v^{\prime}=\frac{\lambda}{\tau} .
$$

So, likewise as in (2.54) and (2.55),

$$
\delta\left(\epsilon^{2}+\text { const }\right)=\delta\left(p^{\prime} v^{\prime} p v\right)
$$

and thus

$$
\epsilon^{2}+\text { const }=p^{\prime} p v^{\prime} v+\text { const }
$$

Next replacing (2.50) in (2.44), one also finds

$$
\frac{1}{2} \delta\left(\epsilon^{2}+\text { const }\right)=n \hbar^{2} \frac{1}{\tau} \delta\left(\frac{1}{t}\right)=n \hbar^{2}\left(\frac{1}{t^{\prime 2}}-\frac{1}{t^{\prime 2}}\right)
$$

i.e., with trivial manipulations,

$$
\epsilon^{2}+\text { const }=2 n \hbar^{2} \frac{t^{\prime \prime 2}-t^{\prime 2}}{\left(t^{\prime} t^{\prime \prime}\right)^{2}}=2 n \hbar^{2} \frac{c^{2} t^{\prime \prime 2}-c^{2} t^{\prime 2}}{\left(c t^{\prime} t^{\prime \prime}\right)^{2}}=2 n(\hbar c)^{2} \frac{c^{2} t^{\prime \prime 2}-c^{2} t^{\prime 2}}{\left(c^{2} t^{\prime} t^{\prime \prime}\right)^{2}} .
$$

Rewrite this last result in two ways, taking into account that either $c^{2} t^{\prime 2}$ or $c^{2} t^{\prime \prime 2}$ can be regarded as a square space range whereas the other one still remains a square time multiplied by $c^{2}$; so

$$
\epsilon^{2}+\text { const }=2 n(\hbar c)^{2} \frac{c^{2} t^{\prime \prime 2}-\delta x^{\prime 2}}{\left(\delta x^{\prime} c t^{\prime \prime}\right)^{2}}, \quad \delta x^{\prime 2}=c^{2} t^{\prime 2}
$$

or

$$
\epsilon^{2}+\text { const }=2 n(\hbar c)^{2} \frac{\delta x^{\prime \prime 2}-c^{2} t^{\prime 2}}{\left(\delta x^{\prime \prime} c t^{\prime}\right)^{2}}, \quad \delta x^{\prime \prime 2}=c^{2} t^{\prime \prime 2} .
$$

Emphasizing again that all quantities at the right hand side are arbitrary, unknown and unknowable because of the uncertainty, one acknowledges three important features of this result written without primed and double primed notations

$$
\delta s^{2}=c^{2} t^{2}-\delta x^{2}, \quad \delta s^{* 2}=\delta x^{2}-c^{2} t^{2} .
$$

If the finite value of $c$ is that of a constant and invariant velocity and these quantities are invariant in different inertial reference systems, then $\varepsilon^{2}$ defined in this way is invariant itself. This requirement is very easy to fulfill, it is enough to replace in (2.55) $v^{\prime} v=c^{2}$ : as $v=v^{\prime}=c$ in this particular case, $\varepsilon^{2}$ depends upon the variable quantity $(p c)^{2}$ only, the constant being invariant by definition. Hence the equation of interest is

$$
\epsilon^{2}=(p c)^{2}+\text { const }^{\prime \prime}, \quad \text { const }{ }^{\prime \prime}=\text { const }{ }^{\prime}-\text { const },
$$


which automatically implies $p$ invariant as well. Note that $\varepsilon^{2}=(p c)^{2}+$ const yields $\varepsilon \delta \varepsilon=c^{2} p \delta p$ and thus

$$
p=\frac{\epsilon}{c^{2}} \frac{\delta \varepsilon}{\delta p}=\frac{\epsilon}{c^{2}} \frac{n \hbar}{\delta t} \frac{\delta x}{n \hbar}=\frac{\epsilon}{c^{2}} v, \quad v=\frac{\delta x}{\delta t}
$$

so that

$$
p=\frac{\epsilon}{c^{2}} v
$$

Hence, if $c$ is an invariant then (2.57), (2.58) define four relativistic invariants $\delta x \delta t,(2.59),(2.60)$ and (2.61). Also note that $p \rightarrow 0$ for $v \rightarrow 0$ but exists the finite limit

$$
\lim _{v \rightarrow 0} \frac{p}{v}=m_{0}=\frac{\epsilon_{0}}{c^{2}}
$$

whereas there is no reason to require that $\varepsilon$ for $v \rightarrow=$ as well; this finite value $m_{0}$ is the rest mass of the particle. Here is at last the mass. So for $p \rightarrow 0$ (2.61) reads

$$
\lim _{v \rightarrow 0} \varepsilon=\varepsilon_{0}=m_{0} c^{2}
$$

Eventually, it is immediate to acknowledge that (2.60) and (2.61) define the Lorentz factor $\sqrt{1-v^{2} / c^{2}}$. Indeed replacing (2.61) into (2.60) one finds

$$
\epsilon^{2}=\frac{\text { const }^{\prime \prime}}{1-v^{2} / c^{2}}, \quad \text { const }^{\prime \prime}=\left(m_{0} c^{2}\right)^{2}
$$

whereas at any $v$ an analogous step yields also

$$
\epsilon^{2}=\frac{\left(m_{0} c^{2}\right)^{2}}{1-v^{2} / c^{2}}
$$

So these equations yield the fundamental achievements of special relativity; the 4-dimensional invariant interval trivially conceivable from this result via the postulated properties of the light speed, in particular, is demonstrated in [16] to be the starting point enough and required to formulate the special relativity.

Also, (2.62) shows how the mass enters explicitly itself into the wave model in a natural and self consistent way, not as a familiar input taken for granted.

But there is more. To conclude this section write now (2.61) as

$$
\epsilon=\mathrm{n} p c, \quad \delta \varepsilon=\delta(\mathrm{n} p c)=c \delta(\mathrm{n} p), \quad \mathrm{n}=\frac{c}{v}
$$

and thus

$$
\frac{\delta \varepsilon}{\delta p}=c \frac{\delta(\mathrm{n} p)}{\delta p}=c \frac{\delta(\mathrm{n} / \lambda)}{\delta(1 / \lambda)}=c \frac{\delta\left(\mathrm{n} v_{0} / \lambda\right)}{\delta\left(v_{0} / \lambda\right)}=c \frac{\delta(\mathrm{n} v)}{\delta(v)}, \quad v=\frac{v_{0}}{\lambda} .
$$

Suppose now that $\delta \varepsilon$ and $\delta p$ are correlated, i.e. $\delta \varepsilon=h v_{g}$ and $\delta p=h / \lambda_{g}$; if $v_{g}$ and $\lambda_{g}$ are not any frequency and wavelength, in which case $\delta \varepsilon / \delta p=v_{g} \lambda_{g} \neq v_{g}$, but rather $v_{g}$ is the velocity of the packet of waves whose energy and momentum are enclosed in $\delta \varepsilon$ and $\delta p$. Let instead $p_{g}$ 
and $\varepsilon_{g}$ be linked by $p_{g}=\varepsilon_{g} v_{g} / c^{2}$; accordingly it is necessary that $\varepsilon_{g}$ and $p_{g}$ fulfill the condition $\delta \varepsilon / \delta p=\varepsilon_{g} / p_{g} \quad$ i.e., owing to (XXX), $\delta \varepsilon / \delta p=c^{2} / v_{g}$. Replacing in (2.62) one finds

$$
v_{g}=\frac{c}{\delta\left(\mathrm{n} v_{g}\right) / \delta v_{g}} .
$$

By implementing the condition (2.61) to calculate $v_{g}$, instead of the phase velocity $\lambda v$, one finds the group velocity of the whole wave packet defined by $\delta \varepsilon$ and $\delta p$.

\subsection{Corpuscular Quantum Mechanics and Special Relativity}

In the previous subsection (2.6) have been implemented to infer the basic equations (2.21) and (2.24) of non-relativistic wave quantum mechanics, then straightforward considerations have shown how even the special relativity is direct consequence of the model of rotating vectors. Now we consider the case where the steps from (2.3) to (2.6) are completely waived, to show that in fact the two (2.1) only are enough themselves to infer all information usually derived solving appropriate wave Equations (2.21) and (2.24) and then the basic equations of special relativity as well. Also, (2.6) are also obtained themselves from (2.1) too.

The invariant equations of the previous section are here inferred as trivial corollary of (2.1) only, writing (2.8) as

$$
\delta \varepsilon=v \delta p, \quad v=\frac{\delta x}{\delta t}=\frac{\delta \varepsilon}{\delta p}
$$

Implement (2.1) taking advantage that a universal constant velocity does in fact exist; so it is convenient to replace $v$ with this constant velocity and write

$$
\delta \varepsilon=\delta(p c),
$$

which means in general

$$
\delta\left(\varepsilon+\text { const }_{\varepsilon}\right)=\delta\left(p c+\text { const }_{p}\right) .
$$

Hence

$$
\varepsilon+\text { const }=p c, \quad \text { const }=\text { const }_{\varepsilon}-\text { const }_{p} .
$$

In principle, being const $_{\varepsilon}$ and const $_{p}$ arbitrary, it is possible that const is positive or negative. Write thus

$$
\varepsilon+\text { const }=p^{\prime \prime} c, \quad \varepsilon-\text { const }=p^{\prime} c .
$$

Merge these equations multiplying them side by side one finds $\varepsilon^{2}=p^{\prime} p^{\prime \prime} c^{2}+$ const $^{2}$ and thus

$$
\varepsilon^{2}=p^{2} c^{2}+\text { const } t^{2}, \quad p^{2}=p^{\prime} p^{\prime \prime} .
$$

Divide this equation by $q \hbar$, being $q$ an arbitrary factor, and note that $\varepsilon / \hbar$ is reciprocal time, $t^{\prime}$, and $p / \hbar$ is reciprocal length, $x^{\prime}$. Then this equation yields 


$$
\frac{1}{\left(q t^{\prime}\right)^{2}}-\frac{v^{2}}{\left(q x^{\prime}\right)^{2}}=\frac{\text { const } / \hbar^{2}}{q^{2}}=\frac{x^{2}-(v t)^{2}}{(x t)^{2}}
$$

being $V$ an arbitrary velocity introduced by dimensional reasons. Since (2.69) is an invariant equation, this result must be invariant as well; this can be obtained simply replacing $v$ with $c$, i.e.

$$
x^{2}-(c t)^{2}=i n v, \quad x t=i n v
$$

as already obtained in (2.59).

Eventually, differentiating (2.71), one finds $c^{2} t \delta t=x \delta x$ and thus, by dimensional reasons,

$$
\frac{c^{2}}{\delta x / \delta t}=\frac{c^{2}}{v}=\frac{x}{t}=\frac{\epsilon}{p}
$$

So

$$
v=p c^{2} / \varepsilon .
$$

This result is sensible; replacing $v$ in (2.67) one finds

$$
\varepsilon \delta \varepsilon=c^{2} p \delta p
$$

i.e., as before,

$$
\delta(\varepsilon)^{2}=\delta(p c)^{2}
$$

and thus again, as in

$$
\varepsilon^{2}=(p c)^{2}+\text { const }
$$

in agreement with (2.69). Eventually calculating the limit $p / v$ for $v \rightarrow 0$ one finds again the rest mass $m_{0}$. Hence also (2.1) yield all invariant quantities and $m$ already found via the wave quantum approach.

Eventually replacing this position in (2.68) one finds, as before,

$$
\varepsilon-\frac{\varepsilon v}{c}=\text { const }, \quad \varepsilon+\frac{\varepsilon v}{c}=\text { const }^{\prime} ;
$$

hence multiplying side by side

$$
\varepsilon\left(1-\frac{v}{c}\right)=\text { const }, \quad \varepsilon^{\prime}\left(1-\frac{\varepsilon v}{c}\right)=\text { const }^{\prime}
$$

one finds

$$
\varepsilon^{2}=\frac{\text { const }{ }^{\prime} \text { const }}{1-v^{2} / c^{2}}
$$

Trivial considerations here omitted for brevity show that const $^{\prime}$ const ${ }^{\prime \prime}=\left(m c^{2}\right)^{2}$. The papers [8] [9] [10] show how to calculate the various constants appearing in these well known equations; it is worth noticing instead that $\left.\varepsilon\right|_{p=0}=m_{0} c^{2}$ implies, in general,

$$
\varepsilon=m c^{2}, \quad m=\frac{m_{0}}{\sqrt{1-v^{2} / c^{2}}} .
$$


It is really remarkable that the results of this section coincide with that of the previous subsections even without involving $\hbar$; indeed $\hbar$ has been introduced in (2.70) for brevity only, it could have been replaced by any other constant with the same physical dimensions. This explains why the wave quantum mechanics is seemingly extraneous to the relativity owing to its evanescent character. However the considerations of the previous section show that the wave approach is essential to demonstrate just the contrary: (2.50), (2.53) and (2.52) are appropriate to obtain the results (2.55) to (2.64). In fact (2.1) are anyway the common conceptual basis of both wave and corpuscular quantum physics and relativity.

\section{Specific Examples of Quantum Systems}

So far have been introduced equations that involve the quantum/relativistic definitions of energy and momentum. Now we concern specifically how these dynamical variables define the quantum systems. To exemplify this idea consider the standard definition of angular momentum $\boldsymbol{M}=\boldsymbol{r} \times \boldsymbol{p}$; actually a more general definition of the vectors at the right hand side is $\boldsymbol{M}^{*}=\left(\boldsymbol{r}_{1}-\boldsymbol{r}_{0}\right) \times\left(\boldsymbol{p}_{1}-\boldsymbol{p}_{0}\right)$, with explicit notation of both coordinates defining their orientation and modulus. In fact (2.1) requires this notation: indeed the range $\boldsymbol{r}_{1}-\boldsymbol{r}_{0}$ allows emphasizing that any random local coordinate $\boldsymbol{r}_{0} \leq \boldsymbol{r} \leq \boldsymbol{r}_{1}$ is included in $\delta \boldsymbol{r}$. Analogous reasoning holds for the random local $\boldsymbol{p}$ defined in $\boldsymbol{p}_{0} \leq \boldsymbol{p} \leq \boldsymbol{p}_{1}$. In classical physics any $r$ is in principle definable and exactly knowable; according to the concept of uncertainty, however, neither $\boldsymbol{r}_{1}$ nor $\boldsymbol{r}_{0}$ and thus $\boldsymbol{r}$ do so in principle. Of course the same holds for the momentum range. This marks the profound difference between classical and quantum physics. Read thus the local coordinate and momentum as random dynamical variables in the respective uncertainty ranges $\delta \boldsymbol{r}$ and $\delta \boldsymbol{p}$; the ranges of dynamical variables, and not their random local values, have actual physical meaning. Consider thus

$$
\boldsymbol{M}^{*}=\delta \boldsymbol{r} \times \delta \boldsymbol{p}
$$

assuming that $\boldsymbol{r}_{0}-\boldsymbol{r}_{0}$ and $\boldsymbol{p}-\boldsymbol{p}_{0}$ are related to their own two-dimensional coordinate/momentum reference system. The fact that both are unknowable in principle, and not as a kind of approximation to simplify some step of the problem, makes indefinable the reference system itself. Now the usual approach to calculate $M=|\boldsymbol{M}|$ is replaced by that exclusively governed by (2.1). Calculate the component of $\boldsymbol{M}^{*}$ along an arbitrary direction defined by the unit vector $\boldsymbol{k}$; so it is possible to write

$$
M_{k}^{*}=(\delta \boldsymbol{r} \times \delta \boldsymbol{p}) \cdot \boldsymbol{k}=(\boldsymbol{k} \times \delta \boldsymbol{r}) \cdot \delta \boldsymbol{p}=\delta \boldsymbol{u} \cdot \delta \boldsymbol{p}, \quad \delta \boldsymbol{u}=(\boldsymbol{k} \times \delta \boldsymbol{r}) .
$$

The scalar product on the right hand side defines thus

$$
\delta p_{u}=\delta \boldsymbol{p} \cdot \frac{\delta \boldsymbol{u}}{|\delta \boldsymbol{u}|},
$$

whereas (3.2) reads

$$
M_{k}^{*}=|\delta \boldsymbol{u}| \cdot \delta p_{u}
$$


Since hold (2.1) also in the case of length range size $|\delta \boldsymbol{u}|$ and conjugate momentum range size $\delta p_{u}$, then

$$
M_{k}^{*}=0 \quad \text { or } M_{k}^{*}= \pm l \hbar, \quad l=\text { integer },
$$

where the integer $n$ of (2.1) has been quoted here with notation $l$ according to the current literature; of course the double sign accounts for the possible signs of the component $M_{k}^{*}$ of $\boldsymbol{M}$ along $\boldsymbol{k}$. Hence the quantum angular component of angular momentum along an arbitrary direction can be 0 or \pm an arbitrary integer times $\hbar$. This component is actually is the only one knowable because repeating the reasoning for a different $\boldsymbol{k}^{\prime}$ would not imply in fact any new physical information.

The space equivalence of three space dimensions agrees with the conclusion that must hold statistically $\left\langle M_{x}^{2}\right\rangle=\left\langle M_{y}^{2}\right\rangle=\left\langle M_{z}^{2}\right\rangle$ compatible with the space isotropy in the absence of external fields. Each average is calculated from two arbitrary integer values $-L$ and $L$ allowed to $I$ as follows

$$
\left\langle M_{k}^{2}\right\rangle=\hbar^{2} \sum_{l_{k}=-L}^{L} \frac{l_{k}^{2}}{2 L+1}=\hbar^{2} \frac{L(L+1)}{3}
$$

whence

$$
M^{2}=\left\langle M_{x}^{2}\right\rangle+\left\langle M_{y}^{2}\right\rangle+\left\langle M_{z}^{2}\right\rangle=\hbar^{2} L(L+1) .
$$

Eventually note that it is possible to write

$$
\frac{M^{2}}{\hbar^{2}}=\left(L+\frac{1}{2}\right)^{2}-\frac{1}{4}
$$

that reads, after summing $L+1 / 2$ at both sides,

$$
\frac{M^{2}}{\hbar^{2}}+\frac{1}{4}+\left(L+\frac{1}{2}\right)=\frac{\mathcal{M}^{2}}{\hbar^{2}}=\left(L+\frac{1}{2}\right)\left[\left(L+\frac{1}{2}\right)+1\right]
$$

and in turn defines a new component $\mathcal{M}_{z}=(L+1 / 2) \hbar$ by comparison with (3.3). The generalization of this result to the case of the spin-orbit coupling $L \pm S$ is concerned in [17]. Note only that in fact $L$ is arbitrary integer; hence, when considering $L^{\prime}=L+1$, then $\mathcal{M}^{2}$ reads $\left(L^{\prime}-1 / 2\right)\left(\left(L^{\prime}-1 / 2\right)+1\right) \hbar^{2}$ whereas $M_{z}=\left(L^{\prime}-1 / 2\right) \hbar$. An analogous way of reasoning would show easily that even $\mathcal{M}_{z}=L \pm S$ with $S=1 / 2,3 / 2, \cdots$ must be in fact possible.

Is evident in this reasoning that the total angular quantum number $L$ implies the existence of a further quantum number $l_{k}$ introduced in (3.4), i.e. magnetic quantum number usually denoted as $\mathrm{m}$, such that $-L \leq \mathrm{m} \leq L$. These results are well known and do not need further comments. It is only worth noticing that, as expected, the steps (2.3) to (2.6) have not been involved; i.e. the quantum angular momentum, just as it is currently acknowledged, has been correctly obtained without implementing the wave formalism.

Introduce now the energy $E=E(\delta x)$ of a system of two particles $\delta x$ apart. A two body system is here proposed in order to simplify and shorten the exposition of results comparable with that already known. Waiving at the mo- 
ment any hypothesis about the kind and analytical form of internal interaction, the most general way to describe $E$ is the sequence of terms

$$
E_{x} \sim \cdots a_{1} \delta x^{2}+a_{2} \delta x+a_{3}+\frac{a_{4}}{\delta x}+\frac{a_{5}}{\delta x^{2}}+\frac{a_{6}}{\delta x^{3}}+\cdots
$$

moreover $E=E(\delta p)$ can be also expanded in series of powers of conjugate moment range $\delta p$ corresponding to quantum state where the two particles are $\delta x$ apart, i.e.

$$
E_{p} \sim \cdots a_{1}^{\prime} \delta p^{2}+a_{2}^{\prime} \delta p+a_{3}^{\prime}+\frac{a_{4}^{\prime}}{\delta p}+\frac{a_{5}^{\prime}}{\delta p^{2}}+\frac{a_{6}^{\prime}}{\delta p^{3}}+\cdots
$$

First of all (3.7) and (3.8) are more than reasonable and general positions: in fact the term $a_{5}^{\prime} / \delta p^{2}$ corresponds to $a_{1} \delta x^{2}$, whereas $a_{2}^{\prime} \delta p$ corresponds to $a_{4} / \delta x$ and so on. The fact that both sequences are admissible and compatible justifies the chance of defining a comprehensive sequence of energy terms that merges $\delta x^{k}$ terms diverging for $\delta x \rightarrow 0$ and for $\delta x \rightarrow \infty$ as well; next the physical problem discriminates itself the appropriate sequence depending on the physical problem, e.g. (3.7) with coefficients $a_{j}=0$ either for $j<3$ or $j>2$. Of course without quantum uncertainty (2.1) the concurrent positions (3.7) and (3.8) would be difficult to explain. In fact the specific kind of problem is the discriminant condition to implement (3.7). To determine the constant coefficients $a_{j}$ of this series expansion of $E$ one could require for example an interaction that vanishes at infinity; this is typically the case of two charges $\delta x$ apart. But it is also possible to think a system whose interaction force increases with $\delta r$, as for example the harmonic oscillator; if an elastic spring tethers two bodies, then the more one stretches the spring the more one increases the internal energy of the system. Are known further examples of the first and second kind, e.g. respectively the gravity force and the strong force between nucleons in the atomic nucleus. In the former case it is intuitive the chance of putting equal to zero the divergent coefficients $a_{j}$ with $j \leq 3$, whereas in the second case are presumably relevant the coefficients $a_{j}$ with $j \geq 3$. Eventually, as a further example, the coefficient $a_{6}$ in (3.7) concerns the Casimir energy. Follow now just a few examples to test and explain (3.7).

Write thus

$$
E_{x}-a_{3}=\delta \varepsilon=a_{4} \frac{\delta p}{n \hbar}+a_{5} \frac{(\delta p)^{2}}{(n \hbar)^{2}}, \quad a_{1}=0, \quad a_{2}=0, \quad a_{j>5}=0
$$

having approximately neglected all other coefficients. Rewrite identically $\delta \varepsilon$ as

$$
\delta \varepsilon=a_{5}\left(\frac{\delta p}{n \hbar}+\frac{a_{4}}{2 a_{5}}\right)^{2}-a_{5}\left(\frac{a_{4}}{2 a_{5}}\right)^{2}:
$$

at this point there are two chances, $a_{4} \neq 0$ or $a_{4}=0$.

1) In the former case (3.9) can take a form of minimum energy requiring the vanishing of the first addend surely positive with $a_{5}>0$, and thus 


$$
\delta x_{\min }=\frac{n \hbar}{\delta p_{\min }}=-\frac{2 a_{5}}{a_{4}}, \quad \delta \varepsilon_{\min }=-a_{5}\left(\frac{a_{4}}{2 a_{5}}\right)^{2}:
$$

in this case $\delta \varepsilon_{\min }<0$ means $E_{x}<a_{3}$, i.e. a bound state corresponding to $a_{4}<0$. To asses this result, note that putting in particular

$$
a_{4}=-Z e^{2}=|Z e| e, \quad a_{5}=\frac{(n \hbar)^{2}}{2 m}
$$

so that

$$
\delta x_{\min }=\frac{(n \hbar)^{2}}{Z e^{2} m}, \quad \delta \varepsilon=E-a_{3}=-\frac{Z^{2} e^{4} m}{2(n \hbar)^{2}} .
$$

one finds the well known expressions of Bohr radius and hydrogenlike energy. Here $\delta \varepsilon$ indicates the range of all energy values $\epsilon_{n}$ allowed by the arbitrary values of the integer $n$, being of course $a_{3} \leq \epsilon_{n} \leq E$. The meaning of quantum number becomes here number of quantum states, by necessity integer. Obviously the positions (3.12) have been necessarily introduced here "ad hoc" once having waived the concepts of Coulomb potential and electromagnetic interaction, only to shorten the exposition; here the aim was to show the physical meaning of the coefficients in (3.7). Yet this topic has been concerned more in detail in several papers [13] [18], which show that $a_{3}$ yields the quantized rotational energy of the atom as a whole. The reason why (3.12) are approximation is evident: all higher order terms of (3.7) have been neglected. Actually this result has been extended even to the case of relativistic one electron atom, thus obtaining the Dirac formula including also the Lamb shift term [13]. This short reasoning aimed merely to show that the coefficients of series expansion (3.8) are not just numbers, rather they have physical meaning related to the energy eigenvalues and Bohr radius usually inferred solving the appropriate energy wave function.

Note that $\delta x_{\min } \delta \varepsilon_{\min }=a_{4}$, i.e. the Coulomb law $\delta \varepsilon_{\min }=a_{4} / \delta x_{\min }$ in the given approximation (3.7) or (3.8) that neglect other powers of $\delta x$ and $\delta p$. Clearly the Coulomb law fulfill this result, i.e. with the choice (3.11) the Bohr energy (3.12) takes the expected form $|Z e| e / 2 \delta x$.

2) However nothing compels just the definitions (3.11), provided that the new physical dimensions are still compliant with meaning of energy of all (3.7) terms. For example even $G m^{2}=G m_{1} m_{2}$ has the same physical dimensions of $e^{2}$, whereas $a_{5}=G \ell m^{2}$ is dimensionally consistent with $(n \hbar)^{2} / m$ if $m_{1}$ and $m_{2}$ are two arbitrary masses, $G$ a constant and $\ell$ an arbitrary length. So putting

$$
a_{4}=-m_{1} m_{2} G, \quad a_{5}=\frac{G \ell m_{1} m_{2}}{2}
$$

and replacing in (3.10), the result compliant with (3.9) and corresponding to (3.12) takes the form

$$
\delta x_{\min }=\ell, \quad \delta \varepsilon_{\min }=-G \frac{m_{1} m_{2}}{\ell} .
$$

On the one hand the series expansions (3.7) and (3.8) explain why Newton 
and Coulomb laws, although both approximations because defined by a restricted number of terms, take an analogous analytical form. On the other hand this means that the constants $e^{2}$ and $G$ should be somehow related, owing their common origin at least in the given approximation. This is in effect true because in c.g.s. system

$$
e=k G, \quad G=6.68 \times 10^{-8} \mathrm{~cm}^{3} / \mathrm{g} \cdot \mathrm{s}^{2}, \quad e=4.80 \times 10^{-10} \text { u.e.s. }
$$

being $k$ a dimensional proportionality constant. Taking $k=k^{\prime} \alpha$, where $k^{\prime}$ is the dimensional factor of $k \equiv \alpha$, this last equation reads in effect from a numerical point of view

$$
4.80 \times 10^{-10}=k^{\prime} \times 4.87 \times 10^{-10} ;
$$

i.e. $k^{\prime}$ is numerically equal to 1 with good approximation. This shows the sought numerical relationship between $e$ and $G$ through the dimensional factor $k^{\prime} \approx 1$.

Note that the result (3.14) is consistent with a different choice of $a_{4}$ and $a_{5}$ in $(3.9)$

$$
a_{4}=-4 \frac{G^{2} m^{3}}{\ell v^{2}}, \quad a_{5}=4 \frac{m^{4} G^{3}}{\ell v^{4}}
$$

being $v$ an arbitrary velocity. These positions seem apparently weird, however a trivial dimensional analysis shows that both are consistent with (3.10) likewise as the previous (3.11). In effect it is immediate to calculate

$$
\delta x_{\min }=-\frac{2 a_{5}}{a_{4}}=\frac{2 m G}{v^{2}}, \quad \delta \varepsilon_{\min }=-\frac{a_{4}^{2}}{4 a_{5}}=-G \frac{m^{2}}{\ell}=-G \frac{m_{1} m_{2}}{\ell} ;
$$

it is clear that being $m$ arbitrary, it can be replaced by the product of $m_{1}$ and $m_{2}$ arbitrary themselves as well. Hence, as expected, the second equation is in fact equivalent to the second (3.14). It is surprising the first equation; the serendipic result is that it yields the escape velocity $v$ from the mass $m$ located $\delta x_{\min }$ apart. So for $v \rightarrow c$ one defines the particular length

$$
\ell_{b h}=\frac{2 m_{1} G}{c^{2}}
$$

below which even the photon cannot longer escape. Equation (3.15), (3.16) and (3.18) show the connection between gravity interaction and electromagnetic interaction.

3) If instead $a_{4}=0$ then (3.9) reads trivially

$$
\delta \varepsilon=a_{5}\left(\frac{\delta p}{n \hbar}\right)^{2}=\frac{(\delta p)^{2}}{2 m}
$$

i.e., likewise as done in (2.52) to (2.61), $\epsilon=p^{2} / 2 m+$ const that is clearly the kinetic energy of the whole atom.

These results suggest in turn how to describe another system with different interaction. Indeed the electromagnetic interaction was implied by the coefficient $a_{4}$ only, without which this result is a mere kinetic term. This means that 
if instead of considering $E_{x}$ via the terms $a_{4}$ and $a_{5}$ one considers, for example, the terms $a_{1}$ and $a_{2}$ along with $a_{5}$ one should concern another kind of system physically sensible. This is highlighted in the next example.

4) Consider again (3.8) noting that

$$
\begin{aligned}
& a_{1} \delta x^{2}+a_{2} \delta x=a_{1}\left(\delta x+\frac{a_{2}}{2 a_{1}}\right)^{2}-a_{1}\left(\frac{a_{2}}{2 a_{1}}\right)^{2}=a_{1} \delta x^{\prime 2}-a_{1}\left(\frac{a_{2}}{2 a_{1}}\right)^{2}, \\
& \delta x^{\prime}=\delta x+\text { const }:
\end{aligned}
$$

in fact considering $\delta x^{\prime}$ instead of $\delta x$ is irrelevant, because the uncertainty does not fix the range sizes, which are instead conceptually unknown and unknowable. So keeping the term with coefficient $a_{5}$ and taking advantage of the fact that the range boundaries of $\delta \varepsilon$ are arbitrary, write (3.7) as

$$
\begin{aligned}
& \delta \varepsilon=E_{x}-a_{3}+a_{1}\left(\frac{a_{2}}{2 a_{1}}\right)^{2}=a_{1} \delta x^{\prime 2}+\frac{\delta p^{2}}{2 m}=a_{1} \frac{\left(n^{\prime} \hbar\right)^{2}}{\delta p^{2}}+\frac{\delta p^{2}}{2 m}, \\
& a_{4}=0 .
\end{aligned}
$$

Does this new equation still represent a possible quantum physical system indeed? It is known that with $a_{1}=K / 2\left(n^{\prime} \hbar\right)^{2}$ this is the classical energy of harmonic oscillator with force constant $K=\omega^{2} m$. Replacing in (3.19) one finds according to $(2.1)$

$$
\delta \varepsilon=\frac{\delta p^{2}}{2 m}+\frac{n^{\prime} \hbar \omega m}{2 \delta p^{2}}, \quad \omega^{2}=\frac{K}{m},
$$

with the second position (3.19) to allow a non-electromagnetic interaction driven system. It is immediate to verify once more that $\delta \varepsilon$ has a minimum as a function of $\delta p$, i.e. with obvious notation

$$
\delta p_{\min }=\sqrt{n^{\prime} \hbar \omega m}, \quad \delta \varepsilon_{\min }=n^{\prime} \hbar \omega .
$$

For $n^{\prime}=0 \quad$ (3.20) yields $\delta \varepsilon_{0}=\delta p_{0}^{2} / 2 m$ whereas $\delta \varepsilon_{\min }=0$, i.e. there are no vibrational states; thus $\delta \varepsilon_{0} \neq 0$ can be nothing else but $\delta \varepsilon_{\min }\left(n^{\prime}=1\right)$. So $\delta \varepsilon_{0}=m \hbar \omega / 2 m=\hbar \omega / 2$. Hence

$$
\delta \varepsilon_{\min }=\frac{\hbar \omega}{2}+n^{\prime} \hbar \omega, \quad a_{1}=\frac{m \omega^{2}}{2}
$$

as it is well known.

The relevant result is that all quantum numbers that characterize the quantum physics, including the spin, are deductible from (2.1) without additional hypotheses. Also, note that now $\omega$ is not introduced with the fundamental meaning characterizing the steps (2.3) to (2.6) that imply the Planck and De Broglie definitions as corollaries; here $\omega$ is mere notation formally summarizing the shortcut ratio $K / m$, yet the concept of mass is still saved in this approach. In other words $K / \mathrm{m}$ is an experimental definition still including the mass, well different from a conceptual renounce of the mass in fact replaced by the fundamental constant $\hbar$. These examples of calculations show that the coefficients in (3.7) and (3.8) are not mere numbers, rather they have a well identifiable physi- 
cal meaning; hence it is reasonable to expect that other phenomena, e.g. the binding energy at nuclear or subnuclear distance, can be described through higher order coefficients.

\subsection{Rotating Vectors and Special Relativity}

Is it possible to highlight a direct connection between the rotating vectors introduced in section 2.2 and the special relativity? The answer should be positive because from the initial (2.9) have been inferred (2.21) and (2.25), from which the invariant equations of special relativity, Equation (2.55) to (AB5) also follow. Yet it is easy to establish the sought direct link considering (2.60), written as $\varepsilon^{2}=\varepsilon^{*} \varepsilon=\left(i p c+m c^{2}\right)\left(i p c-m c^{2}\right)$, and (2.13) i.e.

$$
\begin{aligned}
& \frac{\varepsilon^{*}}{m c^{2}} \frac{\varepsilon}{m c^{2}}=\left(1+i \frac{p c}{m c^{2}}\right)\left(1-i \frac{p c}{m c^{2}}\right), \\
& \frac{\delta \psi}{\psi}=1-\frac{\psi_{x}}{\psi}=i \frac{\psi_{y}}{\psi} .
\end{aligned}
$$

Split therefore the products at left and right side as

$$
\frac{\varepsilon}{m c^{2}}=1-i \frac{p c}{m c^{2}}, \quad \frac{\varepsilon^{*}}{m c^{2}}=1+i \frac{p c}{m c^{2}},
$$

which can be compared with (2.13) via (2.43)

$$
\begin{aligned}
& \left(\frac{\psi_{x}}{\psi}\right)=1-i(\omega t+\text { const })=1-i \omega \delta t_{\omega}, \\
& \left(\frac{\psi_{x}}{\psi}\right)^{*}=1+i(\omega t+\text { const })=1+i \omega \delta t_{\omega} .
\end{aligned}
$$

It is reasonable to guess with the help of (2.28)

$$
\left(\frac{\psi_{x}}{\psi}\right)=\frac{\varepsilon}{m c^{2}}, \quad\left(\frac{\psi_{x}}{\psi}\right)^{*}=\frac{\varepsilon^{*}}{m c^{2}}, \quad \frac{p c}{m c^{2}}=\omega \delta t_{\omega}:
$$

indeed multiplying side by side (3.24) $1+\left(\omega \delta t_{\omega}\right)^{2}$ and comparing with (2.60) $\varepsilon^{2} /\left(m c^{2}\right)^{2}=1+\left(p c / m c^{2}\right)^{2}$ one finds Hence, recalling (2.13) and (2.21) it is also obtained the following chain of equations

$$
\frac{\delta \psi}{\psi}=1-\frac{\varepsilon^{*}}{m c^{2}}=i \frac{p c}{m c^{2}}=i \frac{p n \lambda_{C}}{\hbar}, \quad \lambda_{C}=\frac{\hbar}{m c}
$$

In effect, collecting all these algebraic steps, one finds the momentum eigenfunction

$$
\psi \equiv \psi_{p}=\exp \left( \pm \frac{i}{\hbar} p x\right), \quad x=n \lambda_{C}
$$

expressed via the Compton length $\lambda_{C}$ of $m$. Also, one finds contextually

$$
\begin{aligned}
& \psi_{\varepsilon}=\exp \left(\mp \frac{i}{\hbar}(p v)\left(n \lambda_{C} / v\right)\right)=\exp \left(\mp \frac{i}{\hbar} \varepsilon t\right), \\
& t=\frac{n \lambda_{C}}{v}, \quad \varepsilon=p v .
\end{aligned}
$$


Eventually the last (3.24) yields the sought link between (2.60) and $\omega$ of (2.16)

$$
\omega=\frac{p}{m c \delta t_{\omega}}
$$

the presence of $\omega$ and $c$ emphasizes the connection of quantum theory with the relativity. The fact of having inferred the correct form of energy and momentum eigenfunctions supports the validity of the positions (3.22) and (3.23) to obtain (3.27) via $p c$ and $m c^{2}$.

Define now a new function $\varphi_{c}$ as follows

$$
\varphi_{c}=\frac{\delta\left(x^{2}\right)}{\delta\left(t^{2}\right)}=\frac{x_{2}^{2}-x_{1}^{2}}{t_{2}^{2}-t_{1}^{2}},
$$

which has clearly physical dimensions of square velocity. Note that the right hand side is not to be confused with $v$ as so far defined, i.e. $v^{2}=(\delta x / \delta t)^{2}$ where $\delta x^{2}=\left(x_{2}-x_{1}\right)^{2}$ and $\delta t^{2}=\left(t_{2}-t_{1}\right)^{2}$. If in particular $\varphi_{c}=$ const, then

$$
\varphi_{c} \delta(t)^{2}=\delta\left(\varphi_{c} t\right)^{2}=\delta(x)^{2}
$$

i.e.

$$
\delta\left(\varphi_{c} t^{2}+\operatorname{const}_{\varphi}\right)=\delta\left(x^{2}+\text { const }_{x}\right)
$$

and thus

$$
\varphi_{c} t^{2}-x^{2}=\text { const }_{x}-\text { const }_{\varphi}=\text { const } .
$$

In this case it is appropriate to put $\varphi_{c}=c^{2}$ to obtain

$$
c^{2} t^{2}-x^{2}=\text { const }, \quad \frac{x_{2}^{2}-x_{1}^{2}}{t_{2}^{2}-t_{1}^{2}}=c^{2}
$$

that agrees with the invariant properties of the square space range $\delta s^{2}$ at left hand sides because one has

$$
c^{2} t^{2}-x^{2}=\text { const }=c^{2} t^{\prime 2}-x^{\prime 2}
$$

in primed and unprimed inertial reference systems. Since this result is invariant, then $c^{2} t^{2}-x^{2}=\delta s_{i n v}^{2}$. Multiplying both sides of (3.28) by any invariant quantity, e.g. $x t / x_{0} t_{0}$ with $x_{0} t_{0}=$ const, one infers in general

$$
c^{2} t^{2}-x^{2}=c^{2} t^{\prime 2}-x^{\prime 2} \text {. }
$$

This result is important because the interval invariancy is foundation of special relativity [16]. Consider now fixed the time square range $\delta\left(t^{2}\right)$ whatever it might be: since $\delta\left(x^{2}\right)>0$ implies by definition $\delta\left(x^{2}\right)>(\delta x)^{2}$, it is possible to write

$$
\varphi=\frac{\delta x^{2}}{\delta\left(t^{2}\right)}=\frac{\delta x^{2}}{\delta t^{2}} \frac{\delta t^{2}}{\delta\left(t^{2}\right)}=r c^{2}=v^{2}, \quad r=\frac{\delta t^{2}}{\delta\left(t^{2}\right)}<1
$$

and therefore also 


$$
\delta \varphi=\varphi_{c}-\varphi=c^{2}-v^{2}, \quad \frac{\delta \varphi}{\varphi_{c}}=1-\frac{v^{2}}{c^{2}} .
$$

Yet, at this point this section cannot be closed without a relevant remark that not only confirms further the validity of the positions (3.21) to (3.27) in defining the eigenfunctions (3.25) and (3.26) but also opens the path to some considerations of general relativity shortly sketched in the next section. Replace to this purpose the last (3.24) into (2.60) to find

$$
\frac{\varepsilon^{2}}{\left(m c^{2}\right)^{2}}=\left(\omega \delta t_{\omega}\right)^{2}+1, \quad \text { const } t^{\prime \prime}=\left(m c^{2}\right)^{2}
$$

and note that

$$
\frac{\varepsilon^{2}}{\left(m c^{2}\right)^{2}} \approx\left(\omega \delta t_{\omega}\right)^{2}, \quad \omega \delta t_{\omega} \gg 1
$$

in fact this is possible because according to (3.23) $\delta t_{\omega} \gg 1 / \omega$ it means $t+$ const $/ \omega \gg 1 / \omega$, i.e. $t \gg(1-$ const $) / \omega$; being $t$ and const both arbitrary, the inequality is fulfilled with appropriate values of $t$ or for const $\approx 1$. Hence with this approximation

$$
\frac{\varphi}{c^{2}} \approx \pm \omega \delta t_{\omega}, \quad \varphi=\frac{\varepsilon}{m}
$$

Differentiate now both sides at a given time $t$, i.e. $\delta t_{\omega}$; so, taking the minus sign in (3.32) the last equation reads

$$
\frac{\delta \varphi}{c^{2}} \approx-\frac{\delta \omega}{\omega_{t}}, \quad \omega_{t}=\frac{1}{\delta t_{\omega}},
$$

as it is known. This result could be also obtained more shortly considering (3.14); replacing $m_{1}=\hbar \omega / c^{2}$ one finds

$$
\varepsilon=-G \frac{m_{1} m}{\ell}=-G \frac{m}{\ell} \frac{\hbar \omega}{c^{2}}
$$

so that

$$
\frac{\varepsilon}{m}=\varphi=-\frac{\hbar G}{c^{2}} \frac{\omega}{\ell}
$$

and thus

$$
-\frac{\varphi}{c^{2}}=\frac{\hbar G}{c^{2}} \frac{\omega}{c^{2} \ell}=\frac{\omega}{\omega_{s t}}, \quad \omega_{s t}=\frac{c^{4} \ell}{\hbar G}
$$

whence the well known result

$$
-\frac{\delta \varphi}{c^{2}}=\frac{\delta \omega}{\omega_{s t}}
$$

\subsection{Some Short Accounts of General Relativity}

Examine the physical meaning of the ratio $\ell_{b h} / \ell$ of (3.18) considering two limit cases expected for a photon freely moving through the space time: the photon 
traveling at infinite distance from $m_{1}$ is unperturbed, the photon passing just at distance $\ell=\ell_{b h}$ is trapped by the gravity center. An intermediate case is that where the path of the photon is simply deviated by the field of $m_{1}$, or equivalently by the space time curvature induced by $m_{1}$, whence the importance of investigating the case $0<\ell_{b h} / \ell<1$. First of all regard $\ell_{b h} / \ell$ approximately as the ratio between an arc of circumference and its radius $\ell$; this ratio defines therefore an angle $\phi / 2$ defined by the center of gravity of $m_{1}$. Consider indeed an arbitrary point on the circumference and two arcs at left and right side around the given point: then $\ell_{b h}-\left(-\ell_{b h}\right)$ defines the total angle $\phi$ defined by the counterclockwise displacement $-\phi / 2=-\ell_{b h} / \ell$ and clockwise displacement $\phi / 2=\ell_{b h} / \ell$ of a photon along a total arc of circumference $2 \ell_{b h}$. This is because the uncertainty prevents distinguishing the clockwise motion of the photon $-\infty \rightarrow \infty$ for the counterclockwise motion $\infty \rightarrow-\infty$; in other words $\ell_{b h}$ is compatible with one half delocalization range only of the photon along the circumference. Since the total $\phi=\phi / 2-(-\phi / 2)$ is equal to that formed by the tangents of the boundaries of the total delocalization arc $\ell_{b h}-\left(-\ell_{b h}\right)$, then $\phi$ is the total deflection of a photon traveling at distance $\ell$ from the source $m^{\prime}$ of gravity field. In other words $2 \ell_{b h} / \ell$ accounts for the impossibility of knowing where a photon comes from or goes to. Then

$$
\phi=\frac{4 m^{\prime} G}{\ell c^{2}} .
$$

Apart from the ideal approximation of circular path along the circle osculating a more complex photon route around $m_{1}$ appear here two essential features of the concept of uncertainty: (i) is unphysical the attempt to distinguish whether any range $\delta x$ symbolizes a linear region of space between two arbitrary boundaries or a curved region anyway complicated, (ii) is unphysical the attempt to distinguish two uncertainty ranges according to their specific assignment, e.g. to describe a harmonic oscillator or hydrogen like atom or light beam bending around a gravity center. Also, a further way to obtain (3.33) is as follows. Write (2.1) considering energy per unit mass instead of energy itself. So

$$
\delta \varphi \delta t=\delta v \delta x=\frac{\delta x}{\delta t} \delta x=\frac{n \hbar}{m}
$$

whence

$$
\frac{\delta \varphi}{c^{2}}=\frac{\hbar}{m c^{2}} \delta \omega, \quad \omega=\frac{1}{\delta t}
$$

Then

$$
\omega_{0}=\frac{m c^{2}}{\hbar}, \quad \delta \omega=\omega_{0} \pm \omega
$$

so that, with the minus sign,

$$
\frac{\delta \varphi}{c^{2}}=-\frac{\delta \omega}{\omega_{0}}, \quad \delta \omega=\omega-\omega_{0} .
$$

The results shortly sketched here as a straightforward consequence of the 
quantum uncertainty will be further concerned in the next section too with direct concern to the concept of space time curvature.

\section{Discussion}

The question posed in section 1 after having introduced $\omega$ in (2.5), i.e. what in fact does rotate, has one possible answer only: the rotating vectors introduced in (2.9) are nothing else but representations of quantum particles. This should not be surprising because one among the most promising physical theories, the string theory, regards the particles as vibrating strings. Since "vibrating" reminds necessarily a vibrational frequency, then the aforesaid $\omega$ is not at all a weird concept additional to the known points of view of the modern physics. Simply this paper has shown a different way to find physical implications of something that vibrates, i.e. rotates with cyclical frequency $\omega$.

The starting idea of the present theoretical model was the assumption of $N$ rotating vectors in the complex plane. The fact that the approach has obtained next the properties of quantum matter shows that the vectors are an effective representation of the matter particles. The massless waves introduced by the wave quantum physics were propaedeutic to the masses of the corpuscular quantum physics, in turn propaedeutic of the relativistic physics, clearly concerning massive particles by definition. Appear the in approach so described three poles resulting from the quantum uncertainty, in turn corollary of the quantum definition (2.2) of space time. This approach suggests therefore an amazing hint to the three worlds of Penrose of Figure 1.

It is surprising that the abstract concept of rotating vectors in a complex plane can account for the properties of matter. Nevertheless, the present theoretical model is an actual representation of three worlds of Penrose:

(i) the second (2.8) does not contain explicitly $\hbar$, so everything inferred from this equation bridges the classical physics to the quantum uncertainty;

(ii) the separate relationships $\delta x \delta p=n \hbar$ and $\delta t \delta \varepsilon=n \hbar$ in the first (2.8) plug the ranges of dynamical variables into the quantum world;

(iii) (2.7) plugs both cases (i) and (ii) into the general relativity, as in fact (2.7) is the essence itself of the covariance inherent (2.1). Also, finite $c$ is required in (2.2) in order to obtain (2.1) as a corollary [10].

Anyone who would inquire how (2.1) change in different reference systems, easily acknowledges that actually such a question is out of place. Indeed $\delta x^{\prime} \delta p^{\prime}=n^{\prime} \hbar$ and $\delta x \delta p=n \hbar$ are actually identities even in different reference systems: $n$ and $n$ 'are not specific values, rather they symbolize sets of integers, so to any $n$ of (2.1) corresponds another $n$ ' without changing anything, i.e. the sets are physically indistinguishable like the respective reference systems themselves.

The uncertainty introduced and implemented in its most agnostic form proposes ranges not directly related to the reality because nothing is knowable about them. Moreover Section 2 has remarked that Equation (2.1) move the physical 
concern from the quantum particles, i.e. the physical world, to the phase space, i.e. a mere mental construct; yet this reassignment, physically sensible though, justifies the quantum indistinguishability of identical particles as a corollary and removes the necessity of compelling it as a postulate. So Penroses question could identically concern why Equation (2.1) should be suitable to describe not only quantum but even cosmological events, as in effect it has been proven true [10]. Here let us sketch very shortly an implication of (2.2). Since the physical dimensions of (2.2) are length ${ }^{3} /$ time write

$$
\frac{\hbar G}{c^{2}}=\frac{\delta \ell^{3}}{\delta t}=\frac{\delta \ell^{3} \hbar \delta t}{\hbar \delta t^{2}}=\frac{\delta \ell^{3} \hbar}{\delta t^{2} \delta \varepsilon}=\frac{\hbar}{\eta \delta t^{2}}, \quad \eta=\frac{\delta \varepsilon}{\delta \ell^{3}},
$$

whence

$$
\delta t^{2}=\frac{c^{2}}{\eta G}, \quad \frac{c^{2}}{G}=1.35 \times 10^{27} \mathrm{~kg} / \mathrm{m} .
$$

Test this result identifying $\eta$ with the energy density in the Universe, and putting thus $\delta t$ as the age of the Universe; with $\delta t=4.35 \times 10^{17} \mathrm{~s}$, one finds

$$
\eta=7.13 \times 10^{-9} \mathrm{~J} / \mathrm{m}^{3}, \quad 7.0963 \times 10^{-9} \mathrm{~J} \cdot \mathrm{m}^{-3} \cdot \text { url } .
$$

It is remarkable the agreement between these results; here appears the link between age of the Universe and vacuum energy density [19]. Consequently the equations (2.1) and the definition (2.2) can be regarded in Figure 6 as scientific core of the Platonic world of pure ideas, to which conforms the physical world governed by the space-time uncertainty: they are a sort of boundary condition to which comply the mental and physical worlds. In this frame the quantum world is an intermediate precursor of the physical world: its non-reality and non-locality are natural consequences of the abstract Equation (2.1) together with the quantization, that in fact turns energy and angular momentum to a set of allowed values. Eventually, the experiment turns the unknowable essence of the quantum world into the knowable reality of the physical world: this last step explains why the Penrose mental world is entitled to include the subtle and elusive quantum world. The link with the physical world represents thus the perturbation induced by the observer during the measurement process; otherwise stated, the real world is a figurative representation of the mental world forcedly upset by the attempt of inspecting its pre-existing hidden reality. In this way an abstract idea of the Plato world turns into what we call physical property.

In summary the present model merges the abstract world of ideas of Plato and its antithetic physical world: the former finds its scientific counterpart in the positions (2.6) implemented as sketched in the subsection 2.1, the latter consists of the perturbed reality deprived by the perfection of the pure ideas via the intermediate mental world. In this sense the agnosticism of the uncertainty is not simply a scientific position, but also a possible way to fit the first step of Penroses scheme linking the Platonic and mental worlds; the Popper culture is nothing else but the attempt to investigate and assess the perturbation process and its outcomes, as in fact we construct our knowledge of the physical world just in- 
terpreting its escaping appearance. The relativity enters into the scheme thanks to its double link with the quantum and classical worlds. It has been shown that relativistic Universe consisting of orbiting systems, black holes and so on corresponds to large numbers of states, i.e. large quantum numbers. In effect the relativity, at least as early formulate by Einstein, is basically classical physics; the concept of $4 \mathrm{D}$ covariance is connected to the mental and physical worlds via the respective classical limits $\hbar \rightarrow 0$ on the quantum side and $c \rightarrow \infty$ on the relativistic side. The three worlds are thus linked as in Figure 6. Penrose worried that the weak point of his scheme is the link between abstract ideas and matter; yet the considerations of Section 2, shortly implemented here, shed some light in this respect according to the title of Penroses book [7]: the special and general relativity are the large, the quantum theory is the small, the deep-rooted space-time uncertainty is the underlying the human mind. In fact the impossibility of specifying anything about the uncertainty ranges means that no information is preceding the Equation (2.1); i.e. these equations, being the origin of our knowledge about the physical universe around us, do not allow any earlier form of knowledge able to provide information about their sizes, boundary coordinates, analytical form and so on. If this information would be somehow available, then something else even more fundamental than the Equation (2.1) should be taken into account; yet this chance seems precluded by the fact that, despite their agnostic character, these equations allow inferring both quantum and relativistic outcomes. An example of this statement is given here.

It is worth emphasizing $\delta \dot{p}=-n\left(\hbar / \delta x^{2}\right) \delta \dot{x}$, i.e. according to (2.1) the space time deformation rate $\delta \dot{x}$ corresponds to the force field $\delta \dot{p}$. This holds not

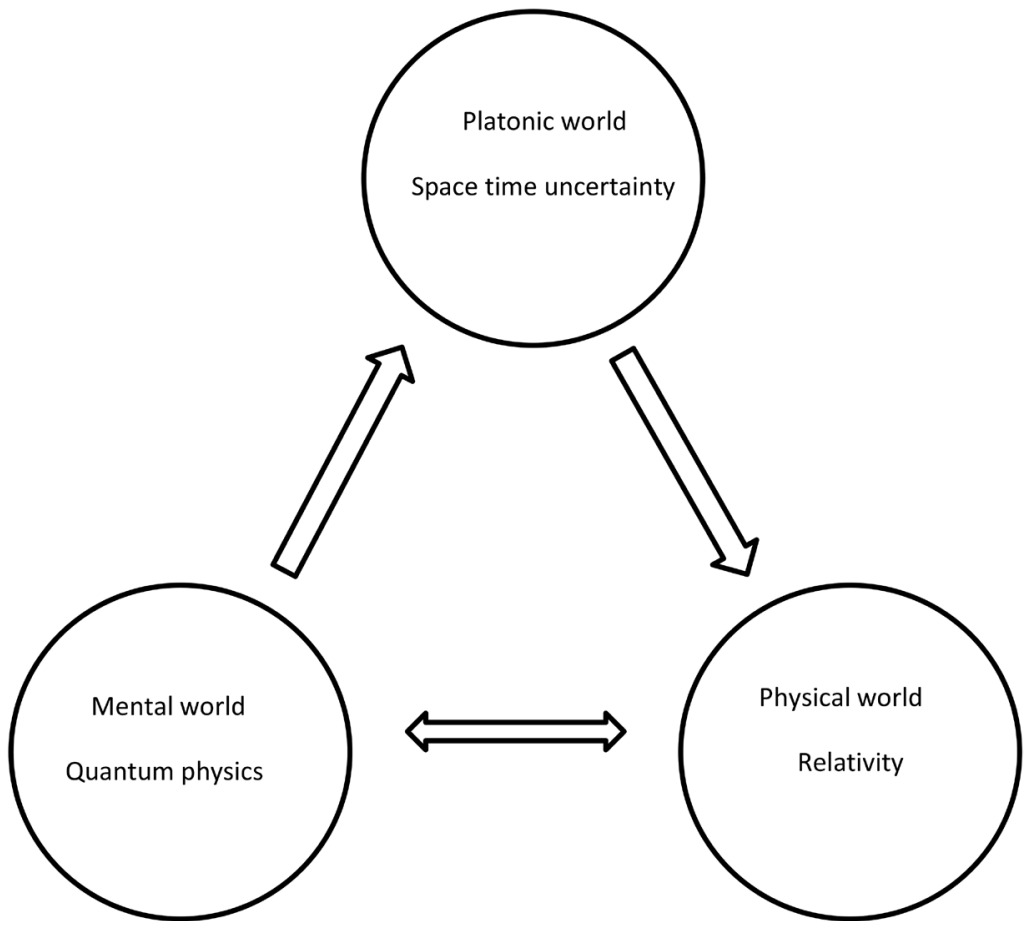

Figure 6. Penrose worlds and their possible connections with worlds of physics. 
only for the gravity, but also for all interaction forces of Nature. In particular the concept of space time curvature is easily recognizable in the present context.

As concerns in this respect the $\delta x^{-1}$ term of the sequence (3.7), follows the next reasoning with the help of an analogous sequence $E_{y}=E_{y}(\delta y)$ of $\delta y$ power terms. It is possible to write

$$
\begin{aligned}
\frac{a}{\delta x}+\frac{b}{\delta y} & =\frac{a \delta p_{x}}{n_{x} \hbar}+\frac{b \delta p_{y}}{n_{y} \hbar}=\frac{n_{x} b \delta p_{y}+n_{y} a \delta p_{x}}{n_{x} n_{y} \hbar} \\
& =\frac{n_{x} \delta p_{y}^{\prime}+n_{y} \delta p_{x}^{\prime}}{n^{2} \hbar}=\frac{n_{x} n_{y}^{\prime} / n^{2} \hbar}{\delta y^{\prime}}+\frac{n_{x}^{\prime} n_{y} / n^{2} \hbar}{\delta x^{\prime}},
\end{aligned}
$$

being $a$ and $b$ appropriate coefficients and

$$
\delta y^{\prime}=b \delta y, \quad \delta x^{\prime}=a \delta x .
$$

Thus

$$
\frac{a}{\delta x}+\frac{b}{\delta y}=\frac{a^{\prime}}{\delta x^{\prime}}+\frac{b^{\prime}}{\delta y^{\prime}}, \quad a^{\prime}=n_{x}^{\prime} n_{y} / n^{2} \hbar, \quad b^{\prime}=n_{x} n_{y}^{\prime} / n^{2} \hbar
$$

Actually $n_{x}$ and $n_{y}$ are not specific values, rather they symbolize sets of integer values; the same of course holds for $n_{x}^{\prime}$ and $n_{y}^{\prime}$, resulting in the algebraic step where $\delta y^{\prime}$ and $\delta x^{\prime}$ are obtained from $\delta p_{y}^{\prime}$ and $\delta p_{x}^{\prime}$. However by dimensional reasons it is possible to write

$$
\frac{a}{\delta x}+\frac{b}{\delta y}=\frac{\xi}{\delta z}=\frac{a^{\prime}}{\delta x^{\prime}}+\frac{b^{\prime}}{\delta y^{\prime}}
$$

being $\delta z$ a third arbitrary range; so that

$$
\frac{a}{\delta x}+\frac{b}{\delta y}=\frac{\xi \delta p_{z}}{n_{z} \hbar}=\frac{a^{\prime}}{\delta x^{\prime}}+\frac{b^{\prime}}{\delta y^{\prime}},
$$

whence

$$
\frac{a}{\delta x}+\frac{b}{\delta y}=\frac{\delta p_{z}^{\prime}}{\delta \varepsilon \delta t}=\frac{a^{\prime}}{\delta x^{\prime}}+\frac{b^{\prime}}{\delta y^{\prime}}, \quad \delta p_{z}^{\prime}=\xi \delta p_{z} .
$$

The result is therefore

$$
\frac{a}{\delta x}+\frac{b}{\delta y}=\frac{F_{z}}{\delta \varepsilon}=\frac{a^{\prime}}{\delta x^{\prime}}+\frac{b^{\prime}}{\delta y^{\prime}}, \quad F_{z}=\frac{\delta p_{z}^{\prime}}{\delta t} .
$$

Compare this result with the standard formula of Laplace curvature, regarding $\delta x$ and $\delta y$ as curvature radii in two arbitrary axes defined in an appropriate primed and unprimed reference systems. These reference systems are actually undefined and conceptually undefinable for two reasons.

On the one hand it is possible to regard the left and right hand sides of (4.1) with a unique physical meaning referred to the aforesaid reference systems.

On the other hand, $\delta x$ and $\delta y$ introduce explicitly the arbitrary lengths $x^{\prime}-x^{\prime \prime}$ and $y^{\prime}-y^{\prime \prime}$ quoting explicitly the upper and lower boundary coordinates, which replace the standard notations $r_{1}$ and $r_{2}$ classically known. The fact that however according to the uncertainty neither of the boundary coordinates is conceptually known and knowable, makes unidentifiable the respective 
primed and unprimed reference systems. In fact, the quantum covariance inherent (2.7) is stronger than Einsteins covariance. The latter takes for granted that different inertial and non-inertial curved reference systems do actually exist, but looks for a unique form of physical equations in any reference system via tensor calculus. The quantum uncertainty excludes instead since the beginning and conceptually the chance of identifying the reference systems themselves in agreement with (2.7). Indeed either boundary coordinate of any uncertainty range, say $x^{\prime \prime}$, could be defined as regards its distance from the origin of the reference system that identifies the location of the range whereas the upper coordinate $x^{\prime}$ should define the range size. Since however neither coordinate is knowable by basic assumption, any reference to the coordinate frame is conceptually inexistent in principle. Nevertheless an observer sitting on $x^{\prime}$ or $x^{\prime \prime}$ experiences different situations when calculating $\delta \dot{x}$ either via $\dot{x}^{\prime}$ with fixed $x^{\prime \prime}$ or via $\dot{x}^{\prime \prime}$ with fixed $\dot{x}^{\prime}$. In both cases the space time deformation $\delta \dot{x}$ implies $\delta \dot{p}$ because of (2.1) and thus the rising of a force field in $\delta x$ : but in one case the observer is at rest with respect to the reference system, whereas in the other case the observer moves solidally with the mobile boundary coordinate he is sitting on. So the observer cannot distinguish whether: (i) he is at rest whereas $\delta \dot{p}$ is due to an external force that stretches or squeezes the range size acting on either boundary coordinate or (ii) $\delta \dot{p}$ the observer is accelerating in a non-inertial reference system. Clearly this is Einsteins equivalence principle, according which no physical difference distinguishes an accelerated reference system or an external gravity force field; in the present context this principle is actually a corollary of the indistinguishability of the aforesaid situations because nothing is knowable about any uncertainty range size. For this reason the present model includes in a natural and self consistent way the extension to the relativistic world, while preserving however the elusive features of the quantum world. The fact that the concept of "distance" is actually unphysical, it would require knowing two points in the space time thus violating (2.1), has significant implications: for example the EPR paradox shouldn't even be formulated, being unphysical the concept of "superluminal" distance itself [20]. Yet, since the curvatures defined in this way are actually non-calculable specifically, it is not surprising that neither the force $F_{z}$ nor the local energy $\varepsilon$ within $\delta \varepsilon$ are in fact definable. It is possible however to say that $F_{z}$ is proportional via $\delta \varepsilon$ to the local curvatures corresponding to all possible random values of $x$ and $y$ falling within $\delta x$ and $\delta y$; so $\delta \varepsilon$, whatever its size might be, is the related range of curvature energies. Once more, likewise as in (3.7) and (3.8), the coefficients a and $b$ characterize the specific cases. For example one recognizes the Laplace-Young capillarity equation in the particular case where $a=b=\gamma A$, being $\gamma$ the surface tension of a liquid and $A$ an arbitrary extent of curved surface, in which case $F_{z} / A$ yields the well known capillary pressure. Also, identifying $\delta \varepsilon$ with $\delta \varepsilon_{\min }$ of (3.14) one infers that the gravity force is directly related to the curvature $\mathcal{R}$ of space time just defined 


$$
F_{z}=\mathcal{R} \delta \varepsilon_{\text {min }}=-G \mathcal{R} \frac{m_{1} m_{2}}{\ell}
$$

To check this result note first that a null curvature implies $F_{z}=0$; moreover it is immediate to infer two further implications. Assuming $m_{1}$ as the source of the gravity field, write

$$
\frac{F_{z}}{m_{2}}=\frac{G \mathcal{R} m_{1}}{\ell}=\frac{\delta s}{\delta t^{2}},
$$

where the second equality is justified by obvious dimensional reasons. This yields the following chain of equations

$$
\frac{G m_{1}}{c^{2} \ell}=\frac{\delta s}{\mathcal{R} c^{2} \delta t^{2}}=\frac{\mathcal{R} \delta s}{(\mathcal{R} c \delta t)^{2}}=\frac{\delta \phi}{4}, \quad \delta \phi=\mathcal{R} \delta s, \quad c \delta t=\frac{2}{\mathcal{R}} .
$$

It is natural to regard $\mathcal{R}$ as reciprocal radius of an ideal sphere and thus $c \delta t$ as an arc of circumference, which in turn defines the angle $\delta \phi$. Also, $c \delta t$ defines a diametral distance $2 / \mathcal{R}$ according to the definition of force per unit mass $F_{z} / m$; indeed the component of a vector force along the direction of the radius defined by $\mathcal{R}^{-1}$ is effective along the whole diameter with respect to the center of mass of $m_{1}$ generating the field, i.e. both opposite radial sizes and not one only. This emphasizes that the force field holds wherever a photon approaches the circumference around the center of gravity at distance $\ell$. Note that $m_{2}$ does not appear explicitly, whereas the fact of having introduced $c \delta t$ and not any $v \delta t$ means that the gravity interaction concerns a moving particle that is just a photon. This yields immediately

$$
\delta \phi=\frac{4 m_{1} G}{c^{2} \ell} .
$$

Moreover write the third equality (4.3) as

$$
\frac{G m_{1}}{c^{2}}=\frac{\mathcal{R} \ell \delta s}{(\mathcal{R} c \delta t)^{2}}=\frac{2 \delta s}{4}, \quad \mathcal{R} \ell=\mathcal{R} c \delta t=2,
$$

having put $\ell=c \delta t$ for the reason just exposed. This yields

$$
2 \frac{G m_{1}}{c^{2}}=\delta s
$$

In fact the concept of gravity force arising because of the mass driven space time curvature is generalized here as space time deformation rate $\delta x$ without any chance of determining what kind of deformation of $\delta x$ is it actually concerned. In effect just this simple corollary of (2.1) is enough to demonstrate the equivalence principles of Einstein [10], thus legitimating the approach to bridge quantum and relativistic worlds in the frame of a unique conceptual approach in a natural and straightforward way. Omitting further details on this point, already published elsewhere, it is interesting the fact that Figure 1 can be integrated by Figure 6. These statements are demonstrated by the ease and immediacy with which have been obtained significant relativistic results. The only constrain in any physical problem is to implement 


\section{$\delta($ dynamical variable $)$}

as done throughout in this paper. Just the uncertainty properties of the ranges justify the chance of defining the derivatives as ratio of two ranges: the tensor formalism concerns by definition local quantities that must be formulated via covariant calculus required "a priori"; here, the covariancy is inherent the uncertainty ranges themselves, and thus does not need any appropriate mathematical formalism.

The fact that appears in (2.48) the ratio $\delta / \delta$ is not merely formal just because of the quantum uncertainty. On the one hand, being the range sizes arbitrary, it can be regarded as $\partial / \partial$ as a limit case with all implications of the standard wave quantum mechanics, see for example the Equation (2.25). Note however that $\partial / \partial$ is uniquely defined by the dependence of the function at numerator upon the dynamical variable at denominator, $\delta / \delta$ is mere ratio of two separate uncertainty ranges that can be handled algebraically as distinct range sizes; only when requiring both sizes tending to zero as a particular case, one introduces a shared property linking in fact the functions at numerator and denominator.

On the other hand, this way of regarding (2.21) and (2.24) allows further implications; in fact (2.42) has been obtained through elementary manipulations of (2.39) where $\delta t$ and $\delta \varepsilon$ can be separately regarded as arbitrary range sizes leading to (2.48). Just this fact, clear consequence of (2.1), emphasizes the role of the statistical formulation of quantum uncertainty in handling $\delta \psi$; in other words $\delta \psi / \delta t$ defines not only the eigenvalues $\varepsilon$, and analogously the momentum eigenvalue as well, but also allows splitting (2.39) as in (2.40) and (2.41). The usual definition of differential $(\partial f / \partial x) \mathrm{d} x$ turns mathematically into $(\delta f / \delta x) \delta x^{\prime}$ assuming tacitly range sizes sufficiently small but still independent; in the latter way even the differentials are consistent with the aforesaid properties of quantum covariance.

The connection between classical and quantum physics is clear once having shown that information resulting from Equation (2.1) is equivalent to that inferred from the solution of wave equations. The boundaries of the uncertainty ranges, e.g. $\varepsilon$ and $\varepsilon_{0}$ of $\delta \varepsilon=\varepsilon-\varepsilon_{0}$ correspond to the classical dynamical variable $\varepsilon$ and its classical boundary condition $\varepsilon_{0}$ due to the initial condition of the specific problem; yet now neither of them is conceptually known or knowable by definition of uncertainty, i.e. the classical values of energy turn into unspecific random values in a given range that in fact take actual physical meaning via (3.7) or (3.8) via the experiment only. Following this idea, even the relativistic results are inferred via a simple mathematical formalism. This conclusion helps to understand the link between the present theoretical model and the relativity, thus justifying Figure 6 .

\section{Conclusions}

The present model has shown that indeed even $\psi$ and $\psi^{*}$ have their own 
physical meaning resulting from the idea of rotating vectors in the complex plane: from (2.10) and (2.11) up to (2.21) and (2.25) following the concepts of $\psi$ and $\psi^{*}$ and then, thanks to (3.22) to (3.24), that of $\psi^{*} \psi$ as well. The Equation (2.9) is indeed a sort of "precursor" of the wave functions, while implying itself well identifiable thermodynamic properties. This conclusion is supported by the essence of Equation (2.31) to (2.38), as if they would concern indeed a lattice of physical particles rather than a collection of abstract rotating vectors.

The results of Section 3.1 and 3.2 show that wave and corpuscular quantum physics are distinct but concurrent topics to infer relativistic results too; the subtle wave/corpuscle dualism of matter, and likewise the quantization itself, do not contradict the relativistic features of the Universe.

\section{Conflicts of Interest}

The author declares no conflicts of interest regarding the publication of this paper.

\section{References}

[1] Wigner, E. (1960) The Unreasonable Effectiveness of Mathematics in the Natural Sciences. John Wiley and Sons, Inc., New York. https://doi.org/10.1002/cpa.3160130102

[2] Russel, B. (1903) The Principles of Mathematics, Vol 1. Cambridge University Press, Cambridge, UK, 404-407.

[3] Penrose, R. (2000) Shadows of the Mind. Random Ed., London.

[4] Penrose, R. (2007) The Road to Reality: A Complete Guide to the Laws of the Universe. A. A. Knopf Ed., New York.

[5] Popper, K. (1972) Epistemology without a Knowing Subject. Oxford University Press, Oxford.

[6] Penrose, R. (2016) Fashion, Faith, and Fantasy in the New Physics of the Universe. Princeton University Press, Princeton. https://doi.org/10.1515/9781400880287

[7] Penrose, R. (1997) The Large, the Small and the Human Mind. Cambridge University Press, Cambridge.

[8] Tosto, S. (1996) An Analysis of States in the Phase Space: The Energy Levels of Quantum Systems. Il Nuovo Cimento B, 111, 193. https://doi.org/10.1007/BF02724645

[9] Tosto, S. (1996) An Analysis of States in the Phase Space: The Diatomic Molecules. Il Nuovo Cimento D, 18, 1363. https://doi.org/10.1007/BF02453780

[10] Tosto, S. (2016) Quantum and Relativistic Corollaries of an Operative Definition of Space Time. Journal of Advances in Physics, 11, 3408. https://doi.org/10.24297/jap.v11i5.362

[11] Polchinski, J. (1998) String Theory. Cambridge University Press, Cambridge. https://doi.org/10.1017/CBO9780511618123

[12] Kiritsis, E. (1998) Introduction to Superstring Theory. Leuven Univ. Press, Leuven.

[13] Tosto, S. (2018) Evolution, Quantization, Relativity: An ab Initio Model. Journal of Modern Physics, 9, 2495. https://doi.org/10.4236/jmp.2018.914161

[14] Tosto, S. (2011) An Analysis of States in the Phase Space: From Quantum Mechan- 
ics to General Relativity.

[15] Bernardini, C. (1979) Fisica e strumenti matematici. Editori Riuniti, Roma, 47. (In Italian)

[16] Landau, L. and Lifchitz, E. (1966) Theorie du Champ. Ed Mir, Moscow. (In French)

[17] Tosto, S. (2017) Many Electron Atoms: Ionization Energies of Trensition Elements. Open Journal of Physical Chemistry, 7, 89-121.

[18] Tosto, S. (2019) Quantum Correlations: Entropy, Wave/Corpuscle Dualism, Bell Inequality. Open Journal of Physical Chemistry, 9, 60-87.

https://doi.org/10.4236/ojpc.2019.92005

[19] https://it.wikipedia.org/wiki/Unit

[20] Tosto, S. (2012) Spooky Action at a Distance or Action at a Spooky Distance? Progress in Physics, 1, 11-26. 